\title{
CONES OF ELEMENTARY IMSETS AND SUPERMODULAR FUNCTIONS: A REVIEW AND SOME NEW RESULTS
}

\author{
Takuya Kashimura \\ Department of Mathematical Informatics, \\ Graduate School of Information Science and Technology, \\ University of Tokyo \\ Tomonari Sei \\ Department of Mathematics, Keio University \\ Akimichi Takemura \\ Department of Mathematical Informatics, \\ Graduate School of Information Science and Technology, \\ University of Tokyo \\ JST, CREST \\ Kentaro Tanaka \\ Department of Industrial Engineering and Management, \\ Graduate School of Decision Science and Technology, \\ Tokyo Institute of Technology
}

\begin{abstract}
In this paper we give a review of the method of imsets introduced by Studeny $1 \frac{11}{1}$ from a geometric point of view. Elementary imsets span a polyhedral cone and its dual cone is the cone of supermodular functions. We review basic facts on the structure of these cones. Then we derive some new results on the following topics: i) extreme rays of the cone of standardized supermodular functions, ii) faces of the cones, iii) small relations among elementary imsets, and iv) some computational results on Markov basis for the toric ideal defined by elementary imsets.
\end{abstract}

\section{Introduction}

The method of imsets by Studeny ${ }^{1}$ provides a very powerful algebraic method for describing conditional independence relations under a probability measure. Rules for deriving conditional independence relations are translated into relations among integer vectors called imsets. Hence many 
properties of conditional independence relations can be conveniently interpreted from a geometric viewpoint. In recent papers Studený and his collaborators $^{2 \mid 3}$ further develop geometric methods for learning Bayesian networks. In this paper we are more concerned on basic geometric properties of imsets, in particular from the viewpoint of lattice bases and Markov bases for the configuration of elementary imsets.

The cone of supermodular functions, which we call the supermodular cone, is defined by a set of linear inequalities and effective inequalities correspond to elementary imsets. Hence the $H$-representation (cf. Grünbaum ${ }^{4}$ ) of the supermodular cone is explicitly given. The cone generated by the elementary imsets, which we call the imset cone, is the dual to the supermodular cone and its set of extreme rays is given by elementary imsets. Hence, in the dual sense, the $V$-representation of the imset cone is given.

From an algorithmic viewpoint of convex geometry, the $V$ representation of the supermodular cone, or equivalently the $H$ representation of the imset cone, are hard to compute and characterize. Therefore general results on the facets of the imset cone, or equivalently the "extreme rays" of the supermodular cone are important. Since the supermodular cone contains a linear subspace consisting of modular functions, we consider extreme rays of the cone of standardized supermodular functions (see (6) below for standardization). These extreme rays are called skeletal supermodular functions in Studený 1

Although the complete description of the supermodular cone and the imset cone is very difficult, some faces of these cones can be studied in detail. In particular a face corresponding to a semi-elementary imset seems to have a simpler structure than other faces.

The organization of the paper is as follows. In Sections 2 and 3 we present a review of the method of imsets from a geometric point of view. In Section 2 we summarize basic facts on the cones of supermodular functions and semi-elementary imsets. In Section 3 we review derivations of conditional independence statements based on multiinformation and imsets. In Section 4 we give some results on extreme rays of the cone of standardized supermodular functions. In Section 5 we discuss properties of a face corresponding to a semi-elementary imset. In Section 6 we characterize relations among a small number of elementary imsets. Finally in Section 7 we discuss some computational results on Markov basis for the toric ideal defined by the configuration of elementary imsets. 


\section{Basic facts on supermodular functions and imsets}

As in Kashimura and Takemura ${ }^{5}$, we use the notation and definitions from Studený $\prod^{1}$ Let $N$ be a finite set and let $\mathcal{P}(N)=\{A: A \subseteq N\}$ denote its power set. In this paper $A \subseteq B$ means that $A$ is a subset of $B$ and $A \subset B$ means that $A$ is a proper subset of $B .|A|$ denotes the cardinality of $A$. For notational convenience, we write the union $A \cup B$ as $A B$. A singleton set $\{i\}$ is simply written as $i . \mathbb{R}, \mathbb{R}^{+}, \mathbb{Q}, \mathbb{Q}^{+}, \mathbb{Z}, \mathbb{Z}^{+}, \mathbb{N}$, denote the sets of reals, non-negative reals, rationals, non-negative rationals, integers, non-negative integers and positive integers, respectively.

$f: \mathcal{P}(N) \rightarrow \mathbb{R}$ is called supermodular if

$$
f(E F)+f(E \cap F) \geq f(E)+f(F), \quad \forall E, F \subseteq N .
$$

The set of supermodular functions over $N$ is denoted by $\mathcal{K}(N)$. $f$ is submodular if $-f$ is supermodular.

$f$ is modular if it is both supermodular and submodular, i.e.

$$
f(E F)+f(E \cap F)=f(E)+f(F), \quad \forall E, F \subseteq N .
$$

$\mathcal{L}(N)=\mathcal{K}(N) \cap(-\mathcal{K}(N))$ denotes the set of modular functions over $N$. A modular function $f$ is like a discrete (signed) measure. Indeed if $f(\emptyset)=0$, then by taking disjoint $E$ and $F$ in (2), we see that $f$ is a measure and hence $f$ can be written as $f(E)=\sum_{e \in E} f(e)$. Without the restriction of $f(\emptyset)=0$, we have $f(E)-f(\emptyset)=\sum_{e \in E}(f(e)-f(\emptyset))$, which can also be written as

$$
f(E)=\lambda_{\emptyset}+\sum_{e \in E} \lambda_{e}, \quad \lambda_{\emptyset}=f(\emptyset), \lambda_{e}=f(e)-f(\emptyset), e \in N .
$$

This shows that the dimension of the linear space $\mathcal{L}(N)$ is $|N|+1$ and a basis of $\mathcal{L}(N)$ is given by the following $|N|+1$ functions:

$$
f_{\emptyset}(E) \equiv 1, \quad f_{e}(E)=1_{e \in \bullet}(E)=1_{e \in E}, \quad e \in N,
$$

where $1_{e \in \bullet}(E)=1_{\{e\} \subseteq \bullet}(E)=1_{e \in E}$ is the indicator function

$$
1_{e \in \bullet}(E)= \begin{cases}1 & e \in E, \\ 0 & \text { otherwise. }\end{cases}
$$

Given a supermodular function $f$, define a modular function $f_{L}$ and a supermodular function $\bar{f}$ by

$$
\begin{aligned}
f_{L}(E) & =\lambda_{\emptyset}+\sum_{e \in E} \lambda_{e}, \quad \lambda_{\emptyset}=f(\emptyset), \quad \lambda_{e}=f(e)-f(\emptyset), \\
\bar{f}(E) & =f(E)-f_{L}(E) .
\end{aligned}
$$


Then $\bar{f}$ is supermodular and $\bar{f}(E)=0$ for $|E| \leq 1 . \bar{f}$ is often a preferred standardization of $f$. By induction on the cardinality of $|E|$, it is easy to show that any standardized supermodular function $f$ is non-negative and non-decreasing:

$$
f(E) \geq 0, \quad E \subseteq F \Rightarrow f(E) \leq f(F), \quad E, F \subseteq N .
$$

Let $N=\{1, \ldots,|N|\}$. We can identify $f: \mathcal{P}(N) \rightarrow \mathbb{R}$ with a vector in $\mathbb{R}^{|\mathcal{P}(N)|}=\mathbb{R}^{2^{|N|}}$ by listing its values:

$$
(f(\emptyset), f(1), \ldots, f(|N|), f(\{1,2\}), \ldots, f(N)) .
$$

With this identification the set of supermodular functions $\mathcal{K}(N)$ is a polyhedral cone in $\mathbb{R}^{2^{|N|}}$, because it is defined by the set of linear inequalities in (11). By the definition of $\mathcal{L}(N)=\mathcal{K}(N) \cap(-\mathcal{K}(N)), \mathcal{L}(N)$ is the largest linear subspace contained in $\mathcal{K}(N)$.

For $A \subseteq N, \delta_{A}: \mathcal{P}(N) \rightarrow \mathbb{R}$ is defined as

$$
\delta_{A}(S)= \begin{cases}1 & \text { if } S=A \\ 0 & \text { otherwise }\end{cases}
$$

For pairwise disjoint subsets, $A, B, C \subseteq N$, we write this triplet by $\langle A, B \mid C\rangle$, and the set of all disjoint triplets $\langle A, B \mid C\rangle$ over $N$ by $\mathcal{T}(N)$. Unless otherwise stated, we assume that $A, B$ are non-empty. On the other hand $C$ may well be an empty set. For a triplet $\langle A, B \mid C\rangle \in \mathcal{T}(N)$, a semielementary imset $u_{\langle A, B \mid C\rangle}: \mathcal{P}(N) \rightarrow \mathbb{R}$ is defined as

$$
u_{\langle A, B \mid C\rangle}=\delta_{A B C}+\delta_{C}-\delta_{A C}-\delta_{B C} .
$$

If $A=\emptyset$ or $B=\emptyset$, then $u_{\langle A, B \mid C\rangle}$ is the zero imset. We consider $u_{\langle A, B \mid C\rangle}$ as a $2^{|N|}$-dimensional integer vector with two elements equal to 1 (at $A B C$ and $C$ ) and two elements equal to -1 (at $A C$ and $B C$ ). If $A=a$ and $B=b$ are singletons, the imset $u_{\langle a, b \mid C\rangle}$ is called elementary. The set of all elementary imsets is denoted by $\mathcal{E}(N)$. Note that the number of elementary imsets is given by

$$
|\mathcal{E}(N)|=\left(\begin{array}{c}
|N| \\
2
\end{array}\right) 2^{|N|-2}=|N|(|N|-1) 2^{|N|-3} .
$$

It is instructive to write out all elementary imsets in a $2^{|N|} \times|\mathcal{E}(N)|$ matrix $\mathcal{U}_{N}$, where each elementary imset is a column of $\mathcal{U}_{N}$. For $|N|=4$, $N=a b c d$, the matrix $\mathcal{U}_{N}$ is written in Table 1 In this paper we consider this matrix as the configuration defining a toric ideal. Let $f(S)=\frac{1}{2}|S|^{2}$, 
Table 1. Configuration of elementary imsets $\mathcal{U}_{N},|N|=4$.

\begin{tabular}{|c|c|c|c|c|c|c|c|c|c|c|c|c|c|c|c|c|c|c|c|c|c|c|c|c|}
\hline & $\begin{array}{l}0 \\
0 \\
0\end{array}$ & $\begin{array}{l}\frac{0}{0} \\
0 \\
0\end{array}$ & $\begin{array}{l}\frac{0}{\sigma} \\
0\end{array}$ & $\begin{array}{l}\frac{\sigma}{0} \\
2\end{array}$ & $\begin{array}{l}\frac{\theta}{\sigma} \\
2\end{array}$ & $\begin{array}{l}\frac{\theta}{\sigma} \\
\dot{v}\end{array}$ & $\begin{array}{l}\frac{\hat{\theta}}{0} \\
\hat{0}\end{array}$ & $\begin{array}{l}\frac{0}{0} \\
\dot{0}\end{array}$ & $\begin{array}{l}\frac{0}{0} \\
0 \\
0\end{array}$ & $\begin{array}{l}\frac{0}{\sigma} \\
\hat{0}\end{array}$ & $\begin{array}{l}\frac{0}{\sigma} \\
\dot{0}\end{array}$ & $\begin{array}{l}\frac{0}{0} \\
0 \\
0\end{array}$ & $\begin{array}{l}\frac{0}{\sigma} \\
0\end{array}$ & $\begin{array}{l}\frac{0}{\sigma} \\
\dot{0}\end{array}$ & $\begin{array}{l}\frac{0}{0} \\
0 \\
0\end{array}$ & $\begin{array}{l}\frac{\sigma}{\sigma} \\
0 \\
0\end{array}$ & $\begin{array}{l}\frac{\sigma}{\sigma} \\
2\end{array}$ & $\begin{array}{l}\frac{\hat{\sigma}}{0} \\
\hat{0}\end{array}$ & $\begin{array}{l}\frac{\theta}{\sigma} \\
\dot{v}\end{array}$ & $\begin{array}{l}\frac{8}{\sigma} \\
2\end{array}$ & $\begin{array}{l}\frac{\theta}{\sigma} \\
\dot{\theta}\end{array}$ & $\begin{array}{l}\frac{0}{0} \\
2\end{array}$ & & 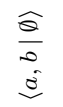 \\
\hline$N$ & 1 & 1 & 1 & 1 & 1 & 1 & 0 & 0 & 0 & 0 & $\overline{0}$ & 0 & 8 & $\overline{0}$ & $\Omega$ & 8 & $\overline{0}$ & $\overline{0}$ & $\overline{0}$ & $\overline{0}$ & $\overline{0}$ & $\overline{0}$ & $\overline{0}$ & \\
\hline$b c d$ & -1 & -1 & 1 & 0 & 0 & 0 & 1 & 0 & 0 & 1 & 0 & & & & & & & & & & & & & \\
\hline & -1 & 0 & 0 & -1 & -1 & c & 0 & & & 0 & & & & & & & & & & & & & & \\
\hline & 0 & -1 & 0 & -1 & 0 & - & 0 & 0 & 1 & 0 & 0 & & & & & & & & & & & & & \\
\hline ao & 0 & 0 & -1 & 0 & -1 & -1 & 0 & 0 & 0 & 0 & & & & & & & & & & & & & & \\
\hline 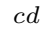 & 1 & 0 & 0 & 0 & 0 & 0 & -1 & -1 & 0 & -1 & -1 & 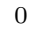 & & & & & & & & & & & & \\
\hline$b c$ & 0 & 1 & 0 & 0 & 0 & & -1 & 0 & -1 & $\rho$ & 0 & & 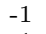 & -1 & & & & & & & & & & \\
\hline & 0 & 0 & & & & & & & & 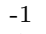 & & & & & -1 & & & & & & & & & \\
\hline$a c$ & 0 & 0 & c & 1 & c & & 0 & -1 & -1 & & & & & & & & & & & & & & & \\
\hline$a$ & 0 & c & & & & & & & & & - & & & & & & & & & & & & & \\
\hline$a b$ & 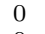 & 0 & c & c & c & & & 0 & 0 & c & 0 & 0 & & -1 & -1 & & 1 & -1 & & & & & & \\
\hline$d$ & 0 & 0 & & & & & & & & & & & & & & & & & -1 & -1 & -1 & & & \\
\hline$c$ & 0 & c & ( & c & & & & & & & & & & & & & & & & & & -1 & -1 & \\
\hline$b$ & 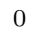 & c & ( & c & & & & & & & & & & & & & & & & -1 & & -1 & & \\
\hline & 0 & & & & & & & & & & & & & & & & & & & & -1 & & -1 & \\
\hline$\emptyset$ & & & & & & & & 0 & U & & 0 & & & & & & & & & & & & & \\
\hline
\end{tabular}

$S \subseteq N$. It is easily seen that this $f$ is supermodular and

$$
\sum_{S \subseteq N} f(S) u(S)=1, \quad \forall u \in \mathcal{E}(N) .
$$

Hence $\mathcal{U}_{N}$ defines a homogeneous toric ideal (Chapter 4 of Sturmfels ${ }^{6}$ ). Note that since $|S|$ is modular, any $f(S)=\frac{1}{2}|S|^{2}+c_{1}|S|+c_{2}, c_{1}, c_{2} \in \mathbb{R}$, satisfies (8). In particular $f(S)=\frac{1}{2}|S|(|S|-1)$ is the standard supermodular function satisfying (8).

In Table 1 rows are ordered (from bottom to top) by the cardinality of the set and by reverse lexicographic order among sets of the same cardinality. We call this order the graded reverse lexicographic order of $\mathcal{P}(N)$. This order can be generalized to elementary imsets as follows. In an elementary imset $u_{\langle a, b \mid C\rangle}$ we always order $a, b$ as $a<b$. Then we define

$$
u_{\langle a, b \mid C\rangle}<u_{\left\langle a^{\prime}, b^{\prime} \mid C^{\prime}\right\rangle} \text { if }\left\{\begin{array}{l}
C<C^{\prime}, \\
\text { or } C=C^{\prime} \text { and } b<b^{\prime}, \\
\text { or } C=C^{\prime} \text { and } b=b^{\prime} \text { and } a<a^{\prime} .
\end{array}\right.
$$

In particular, strict inclusion $C \subset C^{\prime}$ implies $C<C^{\prime}$. For $N=\{1, \ldots,|N|\}$, the maximum element of $\mathcal{E}(N)$ with respect to $<$ is $u_{\langle 1,2 \mid R\rangle}$, where $R=$ $\{3, \ldots,|N|\}$. We call this order the graded reverse lexicographic order of $\mathcal{E}(N)$. This order will be used in the proof of Theorem 6.1 in Section 6 The columns in Table 1 are ordered (from right to left) according to the graded reverse lexicographic order for elementary imsets. 
The following identity among semi-elementary imsets is of basic importance.

$$
u_{\langle A, B D \mid C\rangle}=u_{\langle A, B \mid C\rangle}+u_{\langle A, D \mid B C\rangle} .
$$

This identity can be directly verified as

$$
\begin{aligned}
u_{\langle A, B \mid C\rangle}+u_{\langle A, D \mid B C\rangle}= & \left(\delta_{A B C}+\delta_{C}-\delta_{A C}-\delta_{B C}\right) \\
& \quad+\left(\delta_{A B C D}+\delta_{B C}-\delta_{A B C}-\delta_{B C D}\right) \\
= & \delta_{A B C D}+\delta_{C}-\delta_{A C}-\delta_{B C D} .
\end{aligned}
$$

It is conveniently depicted in Fig. 1.

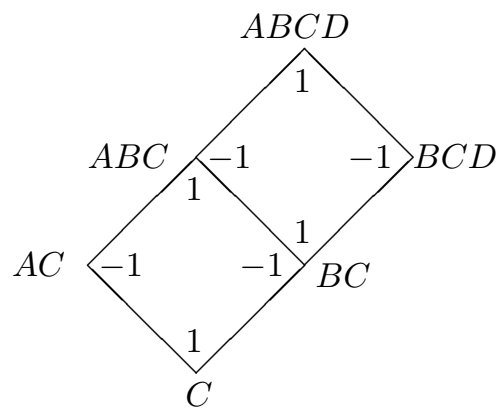

Fig. 1. Sum of two semi-elementary imsets

From (10) we can split $A$ or $B$ in $u_{\langle A, B \mid C\rangle}$ into smaller subsets. If we repeat this splitting, every semi-elementary imset can be written as a nonnegative integer combination of elementary imsets

$$
u_{\langle A, B \mid C\rangle}=\sum_{v \in \mathcal{E}(N)} k_{v} \cdot v, \quad \text { where } k_{v} \in \mathbb{Z}^{+} .
$$

In Kashimura et al ${ }^{7}$ we gave a detailed study of the set of all possible non-negative integer combinations of elementary imsets which are equal to a semi-elementary imset.

We consider $\mathbb{R}^{2^{|N|}}$ as equipped with the standard inner product $\langle\cdot, \cdot\rangle$. Then the inner product of $f: \mathcal{P}(N) \rightarrow \mathbb{R}^{2^{|N|}}$ and $u_{\langle A, B \mid C\rangle}$ is written as

$$
\left\langle f, u_{\langle A, B \mid C\rangle}\right\rangle=f(A B C)-f(C)+f(A C)+f(B C) .
$$

This inner product was already considered in (8). Let $E=A C, F=B C, E \cap$ $F=C, E F=A B C$ in (11). We see that $f$ is supermodular if and only if

$$
\left\langle f, u_{\langle A, B \mid C\rangle}\right\rangle \geq 0, \quad \forall\langle A, B \mid C\rangle \in \mathcal{T}(N) .
$$


Hence the set of semi-elementary imsets $\left\{u_{\langle A, B \mid C\rangle} \mid \forall\langle A, B \mid C\rangle \in \mathcal{T}(N)\right\}$ give the $H$-representation of the supermodular cone $\mathcal{K}(N)$. By definition, the convex cone

$$
\begin{aligned}
\mathcal{K}^{*}(N) & =\mathbb{R}^{+}\left\{u_{\langle A, B \mid C\rangle} \mid\langle A, B \mid C\rangle \in \mathcal{T}(N)\right\} \\
& =\left\{\sum_{\langle A, B \mid C\rangle \in \mathcal{T}(N)} k_{\langle A, B \mid C\rangle} \cdot u_{\langle A, B \mid C\rangle} \mid k_{\langle A, B \mid C\rangle} \geq 0\right\}
\end{aligned}
$$

generated by the semi-elementary imsets is the cone dual to the supermodular cone $\mathcal{K}(N)$. We call $\mathcal{K}^{*}(N)$ the imset cone.

In the $H$-representation of $\mathcal{K}(N)$, not all of the hyperplanes determined by $u_{\langle A, B \mid C\rangle}$ are effective. In fact by (11), if $\langle f, v\rangle \geq 0$ for all elementary $v \in \mathcal{E}(N)$, then $\left\langle f, u_{\langle A, B \mid C\rangle}\right\rangle \geq 0$ for every semi-elementary imset. Hence $f$ is supermodular if $\langle f, v\rangle \geq 0, \forall v \in \mathcal{E}(N)$. It also follows that $\mathcal{K}^{*}(N)$ is generated by the elementary imsets:

$$
\mathcal{K}^{*}(N)=\mathbb{R}^{+} \mathcal{E}(N) .
$$

$\mathcal{K}^{*}(N)$ is a pointed cone by (8). Note that (13) does not yet imply that the every elementary imset is indeed an effective hyperplane defining $\mathcal{K}(N)$, or equivalently every elementary imset is an extreme ray of $\mathcal{K}^{*}(N)$. Note that an extreme ray of a polyhedral convex cone is a half line and when we say " $u$ is an extreme ray of a cone", we actually mean that $\{c u \mid c \geq 0\}$ is an extreme ray of the cone. At this point we establish the following lemma.

Lemma 2.1. Each elementary imset is an extreme ray of the imset cone $\mathcal{K}^{*}(N)$.

There are many ways to prove this lemma. We give a somewhat involved argument, which will be used frequently in Section 6 .

Proof. Let $u=u_{\langle a, b \mid C\rangle} \in \mathcal{E}(N)$ be an elementary imset. Suppose that $u$ is written as a non-negative combination of elements of $\mathcal{K}^{*}(N)$. By (13)

$$
u_{\langle a, b \mid C\rangle}=\sum_{v \in \mathcal{E}(N)} k_{v} \cdot v, \quad k_{v} \geq 0, \forall v \in \mathcal{E}(N) .
$$

We need to show that $k_{v}=0$ for all $v \neq u_{\langle a, b \mid C\rangle}$. Suppose that for $k_{v}>$ 0 for some $v=u_{\left\langle a^{\prime}, b^{\prime} \mid C^{\prime}\right\rangle} \neq u_{\langle a, b \mid C\rangle}$. Among such $v^{\prime}$ s, choose $v_{\max }=$ $u_{\left\langle a^{\prime}, b^{\prime} \mid C^{\prime}\right\rangle}$ such that $a^{\prime} b^{\prime} C^{\prime}$ is the largest according to the graded reverse lexicographic order of $\mathcal{P}(N)$. If $a^{\prime} b^{\prime} C^{\prime}>a b C$, then on the left-hand side of (14) $u_{\langle a, b \mid C\rangle}\left(a^{\prime} b^{\prime} C^{\prime}\right)=0$, but on the right-hand side of (14) $\sum_{v \in \mathcal{E}(N)} k_{v}$. $v\left(a^{\prime} b^{\prime} C^{\prime}\right)>0$. This is a contradiction. Hence if $k_{v}>0$, then $a^{\prime} b^{\prime} C^{\prime} \leq a b C$. 
Similarly, considering the graded reverse lexicographic order of $C$, we have $C^{\prime} \geq C$, if $k_{v}>0$ for $v=u_{\left\langle a^{\prime}, b^{\prime} \mid C^{\prime}\right\rangle}$. In particular for $v=u_{\left\langle a^{\prime}, b^{\prime} \mid C^{\prime}\right\rangle}$ with $k_{v}>0$ we have $|C| \leq\left|C^{\prime}\right|,\left|a^{\prime} b^{\prime} C^{\prime}\right| \leq|a b C|$. But this implies $|C|=\left|C^{\prime}\right|$. Then for any $S$ such that $|S|=|C|$ we have $\sum_{v \in \mathcal{E}(N)} k_{v} \cdot v(S) \geq 0$. Since $u_{\langle a, b \mid C\rangle}(S)=0$ for $S \neq C$, we have $k_{v}=0$ for $v=u_{\left\langle a^{\prime}, b^{\prime} \mid S\right\rangle}, S \neq C$, $|S|=|C|$. Similarly $k_{v}=0$ for $v=u_{\left\langle a^{\prime}, b^{\prime} \mid C^{\prime}\right\rangle}, a^{\prime} b^{\prime} C^{\prime} \neq a b C,\left|C^{\prime}\right|=|C|$. Therefore $k_{v}>0$ only if $C^{\prime}=C$ and $a^{\prime} b^{\prime} C^{\prime}=a b C$. However this implies $v=u_{\langle a, b \mid C\rangle}$.

Let $\mathcal{L}^{*}(N) \subseteq \mathbb{R}^{2^{|N|}}$ denote the linear subspace spanned by the semielementary imsets. $\mathcal{L}^{*}(N)$ is the orthogonal complement of $\mathcal{L}(N)$ and its dimension is

$$
\operatorname{dim} \mathcal{L}^{*}(N)=2^{|N|}-|N|-1 .
$$

$\mathcal{K}^{*}(N) \subset \mathcal{L}^{*}(N)$ and the dimension of the relative interior of $\mathcal{K}^{*}(N)$ is the same as $\operatorname{dim} \mathcal{L}^{*}(N)$. By (4),

$$
u \in \mathcal{L}^{*}(N) \Leftrightarrow \sum_{E \in \mathcal{P}(N)} u(E)=0, \quad \sum_{e \in E \in \mathcal{P}(N)} u(E)=0, \forall e \in N .
$$

Now let

$$
\begin{aligned}
\mathcal{L}_{\mathbb{Z}}^{*} & =\mathbb{Z}\left\{u_{\langle A, B \mid C\rangle} \mid\langle A, B \mid C\rangle \in \mathcal{T}(N)\right\} \\
& =\left\{\sum_{\langle A, B \mid C\rangle \in \mathcal{T}(N)} k_{\langle A, B \mid C\rangle} \cdot u_{\langle A, B \mid C\rangle} \mid k_{\langle A, B \mid C\rangle} \in \mathbb{Z}\right\} \subseteq \mathbb{Z}^{2^{|N|}}
\end{aligned}
$$

denote the submodule of $\mathbb{Z}^{2^{|N|}}$ generated by the semi-elementary imsets. Again by (11), $\mathcal{L}_{\mathbb{Z}}^{*}$ is generated by the elementary imsets:

$$
\mathcal{L}_{\mathbb{Z}}^{*}=\mathbb{Z} \mathcal{E}(N)=\left\{\sum_{v \in \mathcal{E}(N)} k_{v} \cdot v \mid k_{v} \in \mathbb{Z}\right\}
$$

$\mathcal{L}_{\mathbb{Z}}^{*}$ coincides with the set of integer points in $\mathcal{L}^{*}(N)$ :

$$
\mathcal{L}_{\mathbb{Z}}^{*}=\mathcal{L}^{*}(N) \cap \mathbb{Z}^{2^{|N|}}
$$

This can be seen as follows. For each $C \subseteq N,|C| \leq|N|-2$, choose an elementary imset $u_{\langle a, b \mid C\rangle}$. Consider a submatrix of the configuration consisting these elementary imsets, which is of size $2^{|N|} \times\left(2^{|N|}-|N|-1\right)$. If $C^{\prime}$ 's are ordered by the graded reverse lexicographic order as in Table 1, then the lower $\left(2^{|N|}-|N|-1\right) \times\left(2^{|N|}-|N|-1\right)$ part of the matrix is an upper triangular matrix with 1 on the diagonal, as seen in Table 1. Therefore it is unimodular (= has integral inversion). Then the projection of $\mathcal{L}_{\mathbb{Z}}^{*}$ onto 
these $2^{|N|}-|N|-1$ coordinates coincides with $\mathbb{Z}^{2^{|N|}-|N|-1}$. Then (16) holds, because other elements are determined by orthogonality to $\mathcal{L}(N)$.

Let $\mathbb{Q}^{+} \mathcal{E}(N)$ denote the set of non-negative rational combinations of elementary imsets. An integer point in $\mathbb{Q}^{+} \mathcal{E}(N)$ is called a structural imset. Let $\mathcal{S}(N)$ denote the set of structural imsets. Since $\mathcal{K}(N)$ and $\mathcal{K}(N)^{*}$ are rational polyhedral cones, we have

$$
\mathcal{S}(N)=\mathcal{K}^{*}(N) \cap \mathbb{Z}^{2^{|N|}} .
$$

By (16), $\mathcal{S}(N)$ can also be written as $\mathcal{S}(N)=\mathcal{K}^{*}(N) \cap \mathcal{L}_{\mathbb{Z}}^{*}$.

A non-negative integer combination of elementary imsets is called a combinatorial imset and

$$
\mathcal{C}(N)=\mathbb{Z}^{+} \mathcal{E}(N)=\left\{\sum_{v \in \mathcal{E}(N)} k_{v} \cdot v \mid k_{v} \in \mathbb{Z}^{+}\right\}
$$

denotes the set of combinatorial imsets. $\mathcal{C}(N)$ is the semigroup generated by $\mathcal{E}(N)$. Clearly $\mathcal{C}(N) \subseteq \mathcal{S}(N)$ and it is known that for $|N| \geq 5$ the inclusion is strict (Hemmecke et al ${ }^{[}$), i.e. for $|N| \geq 5$ the $\operatorname{semigroup~} \mathcal{C}(N)$ is not normal.

\section{Multiinformation and derivation of conditional independence using imsets}

Let $P, \mu$ be two probability measures on a sample space $\mathcal{X}$, such that $P$ is absolutely continuous with respect to $\mu$. The relative entropy $H(P \mid \mu)$ of $P$ with respect to $\mu$ is defined by

$$
H(P \mid \mu)=\int_{\mathcal{X}} \ln \frac{\mathrm{d} P}{\mathrm{~d} \mu}(x) \mathrm{d} P(x) .
$$

$H(P \mid \mu) \geq 0$ and $H(P \mid \mu)=0$ if and only if $P=\mu$.

Consider a joint probability distribution $P$ of the variables in $N$. As usual, $A \Perp B \mid C[P]$ denotes the conditional independence statement of variables in $A$ and in $B$ given the variables in $C$ under $P$. The set of conditional independences under $P$ is denoted by

$$
\mathcal{M}_{P}=\{\langle A, B \mid C\rangle \in \mathcal{T}(N)|A \Perp B| C[P]\} .
$$

We call $\mathcal{M}_{P}$ the conditional independence model of $P$.

For $A \subseteq N, P^{A}$ denotes the marginal distribution of the variables in $A$. Let $\prod_{i \in A} P^{\{i\}}$ denote the product of one-dimensional marginal distributions in $A$. Then the multiinformation function $m_{P}: \mathcal{P}(N) \rightarrow[0, \infty]$ is 
defined by

$$
m_{P}(S)=H\left(P^{S} \mid \prod_{i \in S} P^{\{i\}}\right), S \neq \emptyset
$$

and $m_{P}(\emptyset)=0$. Throughout this paper we only consider $P$ such that $m_{P}(S)$ is finite for every $S \subseteq N$. The basic fact on the multiinformation is that $m_{P}$ is supermodular. Furthermore the following equivalence between the conditional independence $A \Perp B \mid C[P]$ and the local modularity of $m_{P}$ at $\langle A, B \mid C\rangle$ holds:

$$
\begin{aligned}
\left\langle m_{P}, u_{\langle A, B \mid C\rangle}\right\rangle & \geq 0, \quad \forall\langle A, B \mid C\rangle \in \mathcal{T}(N) \\
& =0 \quad \Leftrightarrow \quad A \Perp B \mid C[P] .
\end{aligned}
$$

This equivalence is the basis for manipulating conditional independence statements in terms of imsets. Note that $m_{P}$ is standardized as a supermodular function since $m_{P}(S)=0$ for $|S| \leq 1$.

Traditionally, the implications among conditional independence statements under a probability measure $P$ have been studied in terms of the following semi-graphoid axioms. In the axioms $A, B, C, D \subseteq N$ are disjoint.

1. triviality $\quad A \Perp \emptyset \mid C[P]$

2. symmetry $A \Perp B|C[P] \Rightarrow B \Perp A| C[P]$

3. decomposition $A \Perp B D|C[P] \Rightarrow A \Perp B| C[P]$

4. weak union $A \Perp B D|C[P] \Rightarrow A \Perp D| B C[P]$

5. contraction $A \Perp D \mid B C[P]$ and $A \Perp B|C[P] \Rightarrow A \Perp B D| C[P]$

Note that decomposition, weak union and contraction can be combined into the following single equivalence:

$$
A \Perp B D|C[P] \quad \Leftrightarrow \quad A \Perp D| B C[P] \text { and } A \Perp B \mid C[P] .
$$

This equivalence can be proved very easily by imsets. Take the inner product of (10) with the multiinformation $m_{P}$. Then

$$
\left\langle m_{P}, u_{\langle A, B D \mid C\rangle}\right\rangle=\left\langle m_{P}, u_{\langle A, B \mid C\rangle}\right\rangle+\left\langle m_{P}, u_{\langle A, D \mid B C\rangle}\right\rangle .
$$

Since every term is non-negative, we have

$$
\begin{aligned}
A \Perp B D \mid C[P] & \Leftrightarrow\left\langle m_{P}, u_{\langle A, B D \mid C\rangle}\right\rangle=0 \\
& \Leftrightarrow\left\langle m_{P}, u_{\langle A, B \mid C\rangle}\right\rangle=\left\langle m_{P}, u_{\langle A, D \mid B C\rangle}\right\rangle=0 \\
& \Leftrightarrow A \Perp D \mid B C[P] \text { and } A \Perp B \mid C[P] .
\end{aligned}
$$

In this way, manipulation of "rules" such as the semi-graphoid axioms is translated to linear algebraic operations in terms of imsets. This is a very important advantage of the method of imsets and the multiinformation. 
As another example, consider the following identity.

$$
\begin{aligned}
u_{\langle a, b \mid c\rangle}+u_{\langle a, c \mid d\rangle}+u_{\langle a, d \mid b\rangle}= & \left(\delta_{a b c}+\delta_{c}-\delta_{a c}-\delta_{b c}\right)+\left(\delta_{a c d}+\delta_{d}-\delta_{a d}-\delta_{c d}\right) \\
& +\left(\delta_{a b d}+\delta_{b}-\delta_{a b}-\delta_{b d}\right) \\
= & \left(\delta_{a b c}+\delta_{a c d}+\delta_{a b d}\right)+\left(\delta_{b}+\delta_{c}+\delta_{d}\right) \\
& -\left(\delta_{a b}+\delta_{a c}+\delta_{a d}\right)-\left(\delta_{b c}+\delta_{b d}+\delta_{c d}\right) \\
= & u_{\langle a, c \mid b\rangle}+u_{\langle a, d \mid c\rangle}+u_{\langle a, b \mid d\rangle} .
\end{aligned}
$$

By the method of imsets we then have

$$
a \Perp b|c, a \Perp c| d, a \Perp d|b \Leftrightarrow a \Perp c| b, a \Perp d|c, a \Perp b| d,
$$

where for simplicity we wrote $a \Perp b \mid c$ instead of $a \Perp b \mid c[P]$. It is evident that none of decomposition, weak union and contraction can be applied to the left-hand side nor to the right-hand side of this equivalence. Therefore this equivalence can not be derived from semi-graphoid axioms.

Here it is interesting to note that (19) can be derived by linear algebraic operations from (10), which itself corresponds to the semi-graphoid axiom. By applying (10) twice we can write

$$
\begin{aligned}
3 \cdot u_{\langle a, b c d \mid \emptyset\rangle}= & \left(u_{\langle a, b \mid \emptyset\rangle}+u_{\langle a, c \mid b\rangle}+u_{\langle a, d \mid b c\rangle}\right) \\
& +\left(u_{\langle a, c \mid \emptyset\rangle}+u_{\langle a, d \mid c\rangle}+u_{\langle a, b \mid c d\rangle}\right) \\
& +\left(u_{\langle a, d \mid \emptyset\rangle}+u_{\langle a, b \mid d\rangle}+u_{\langle a, c \mid b d\rangle}\right)
\end{aligned}
$$

as well as

$$
\begin{aligned}
3 \cdot u_{\langle a, b c d \mid \emptyset\rangle}= & \left(u_{\langle a, b \mid \emptyset\rangle}+u_{\langle a, d \mid b\rangle}+u_{\langle a, c \mid b d\rangle}\right) \\
& +\left(u_{\langle a, c \mid \emptyset\rangle}+u_{\langle a, b \mid c\rangle}+u_{\langle a, d \mid b c\rangle}\right) \\
& +\left(u_{\langle a, d \mid \emptyset\rangle}+u_{\langle a, c \mid d\rangle}+u_{\langle a, b \mid c d\rangle}\right) .
\end{aligned}
$$

Equality of right-hand sides of (21) and (22) immediately gives (19).

Let $u \in \mathcal{S}(N)$ be a structural imset. A conditional independence with respect to $u$ is defined as follows. We say that $A$ and $B$ are conditionally independent given $C$ with respect to $u$, and denote it by $A \Perp B \mid C[u]$, if

$$
\exists k \in \mathbb{N}, k \cdot u-u_{\langle A, B \mid C\rangle} \in \mathcal{S}(N) .
$$

The set of conditional independences induced by $u$ is denoted by

$$
\mathcal{M}_{u}=\{\langle A, B \mid C\rangle \in \mathcal{T}(N)|A \Perp B| C[u]\} .
$$

We call $\mathcal{M}_{u}$ the conditional independence model of $u$. The importance of this definition lies in the following completeness theorem for imsets. 
Theorem 3.1 (Theorem 5.2 of Studený1 ${ }^{1}$ ). Let $P$ be a probability measure over $N$ with finite multiinformation. Then there exists $u \in \mathcal{S}(N)$ such that $\mathcal{M}_{P}=\mathcal{M}_{u}$.

Proof. Define $u=\sum_{\langle A, B \mid C\rangle \in \mathcal{M}_{P}} u_{\langle A, B \mid C\rangle}$. Then clearly $\mathcal{M}_{P} \subseteq \mathcal{M}_{u}$. Now for any $\langle A, B \mid C\rangle \in \mathcal{M}_{u}$ choose $k$ such that (23) holds. Then

$$
0=\left\langle m_{P}, k \cdot u\right\rangle=\left\langle m_{P}, k \cdot u-u_{\langle A, B \mid C\rangle}\right\rangle+\left\langle m_{P}, u_{\langle A, B \mid C\rangle}\right\rangle .
$$

Since two terms on the right-hand side are non-negative, we have $\left\langle m_{P}, u_{\langle A, B \mid C\rangle}\right\rangle=0$ and hence $\mathcal{M}_{u} \subseteq \mathcal{M}_{P}$.

We have reproduced this proof from Section 5 of Studeny ${ }^{11}$, because this argument is instructive. In this proof, to show $\left\langle m_{P}, u_{\langle A, B \mid C\rangle}\right\rangle=0$, we have added an extra term $k \cdot u-u_{\langle A, B \mid C\rangle}$ to $u_{\langle A, B \mid C\rangle}$. In this sense, this proof is similar to the argument concerning (21) and (22) above for showing (20).

Next we show that the conditional independence model $\mathcal{M}_{u}$ depends only on the face of $\mathcal{K}^{*}(N)$ which contains $u$ as a relatively interior point. Although this fact is discussed in a series of papers by Studeny $y^{90}$ and Chapter 5 of Studeny $y^{1}$, we make this point clear in the following lemma.

Lemma 3.1. Let $a_{1}, \ldots, a_{n}$ be nonzero vectors in $\mathbb{R}^{m}$. Let $C=$ $\mathbb{R}^{+}\left\{a_{1}, \ldots, a_{n}\right\}$ be the convex cone generated by these vectors and suppose that $C$ is pointed and $a_{1}, \ldots, a_{n}$ are extreme rays of $C$. For a face $F$ of $C$, let $F^{\circ}$ denote its relative interior. Put $I_{F}=\left\{i \mid a_{i} \in F\right\}$. Then, $b \in C$ belongs to $F^{\circ}$ if and only if the following two conditions hold:

(1) For every $i \in I_{F}$, there exists $k_{i} \in \mathbb{N}$ such that $k_{i} \cdot b-a_{i} \in C$,

(2) For every $i \notin I_{F}$ and every $k \in \mathbb{N}, k \cdot b-a_{i} \notin C$.

Proof. $b \in C$ can be written as $b=\sum_{i=1}^{n} c_{i} a_{i}, c_{i} \geq 0, i=1, \ldots, n$. Suppose that $b$ satisfies the above two conditions. Then for $i \in I_{F}$, by condition 1 , for some $k_{i} \in \mathbb{N}, k_{i} b=a_{i}+\sum_{j=1}^{n} \tilde{c}_{j}^{i} a_{j}, \tilde{c}_{j}^{i} \geq 0$, or

$$
b=\frac{1}{k_{i}} a_{i}+\sum_{j=1}^{n}\left(\tilde{c}_{j}^{i} / k_{i}\right) a_{j}
$$

Taking the average of the right-hand side over $i \in I_{F}$, we see that we can take $c_{i}>0$ for $i \in I_{F}$ in $b=\sum_{i=1}^{n} c_{i} a_{i}$. On the other hand, for $i \notin I_{F}$, condition 2 implies $k \cdot c_{i}-1 \leq 0$ for any $k \in \mathbb{N}$, hence $c_{i}=0$. Therefore if $b$ satisfies two conditions then $b$ is written as

$$
b=\sum_{i \in I_{F}} c_{i} a_{i}, \quad c_{i}>0, i \in I_{F} .
$$


Since the coefficients are positive, we have $b \in F^{\circ}$.

Conversely let $b \in F^{\circ}$. For $i \in I_{F}$ and sufficiently large $k \in \mathbb{N}$, let $a=$ $(1 / k) a_{i}$. Then we have $b-a \in F$, which is equivalent to $k \cdot b-a_{i} \in F \subseteq C$. Let $v \in \mathbb{R}^{m}$ be a normal vector of a supporting hyperplane of $F$ such that $\langle v, x\rangle \geq 0, \forall x \in C$, and for $b \in F$ and any $a_{i} \notin F$ the inner product with respect to $v$ satisfy

$$
\langle v, b\rangle=0, \quad\left\langle v, a_{i}\right\rangle>0 .
$$

Then for any $k \in \mathbb{N}$, we have

$$
\left\langle v, k \cdot b-a_{i}\right\rangle<0,
$$

which means that $k \cdot b-a_{i} \notin C$.

Under the assumptions of Lemma 3.1, let $b \in C$ and let $F=F_{b}$ denote the face of $C$ such that $b \in F^{\circ}$. Let

$$
\mathcal{E}_{b}=\left\{a_{i} \mid k \cdot b-a_{i} \in C, \exists k \in \mathbb{N}\right\} .
$$

Then $\mathcal{E}_{b}$ is the set of extreme rays of $F_{b}$ by Lemma 3.1

From this fact we can prove that conditional independence structures induced by imsets depend only on faces of the imset cone $\mathcal{K}^{*}(N)$ and not on each imset.

Proposition 3.1. Let $u, u^{\prime} \in \mathcal{S}(N)$. Then $\mathcal{M}_{u}=\mathcal{M}_{u^{\prime}}$ if and only if $u, u^{\prime}$ belong to the relative interior of the same face of $\mathcal{K}^{*}(N)$.

Proof. For $v \in \mathcal{S}(N)$, let $F_{v}$ denote the face of $\mathcal{K}^{*}(N)$ such that $v$ belongs to the relative interior $F_{v}^{\circ}$ of $F_{v}$ and let $\mathcal{E}_{v}(N)$ denote the set of elementary imset $u_{\langle a, b \mid C\rangle}$ such that $\exists k \in \mathbb{N}, k \cdot v-u_{\langle a, b \mid C\rangle} \in \mathcal{S}(N)$, that is,

$$
\mathcal{E}_{v}(N)=\left\{u_{\langle a, b \mid C\rangle} \mid\langle a, b \mid C\rangle \in \mathcal{M}_{u}\right\} .
$$

As note above, $\mathcal{E}_{v}(N)$ is the set of extreme rays of $F_{v}$. Hence

$$
\begin{aligned}
\mathcal{M}_{u}=\mathcal{M}_{u^{\prime}} & \Leftrightarrow \mathcal{E}_{u}(N)=\mathcal{E}_{u^{\prime}}(N) \\
& \Leftrightarrow F_{u}=F_{u^{\prime}} .
\end{aligned}
$$

By Theorem 3.1 and Proposition 3.1, each conditional independence model $\mathcal{M}_{P}$ corresponds to a face of $\mathcal{K}^{*}(N)$. Note that, by mutual orthogonality, the face poset of $\mathcal{K}(N)$ and that of $\mathcal{K}^{*}(N)$ are isomorphic (when inclusion in $\mathcal{K}(N)$ is reversed). Hence each conditional independence model $\mathcal{M}_{P}$ also corresponds to a face of $\mathcal{K}(N)$. 
Also note that we can regard the set of conditional independence models $\mathcal{M}_{P}$ as a poset with respect to the inclusion relation $\mathcal{M}_{P} \subseteq \mathcal{M}_{P^{\prime}}$. By Proposition 3.1 this poset is a sub-poset of the face poset of $\mathcal{K}^{*}(N)$. Unfortunately it is known that for $|N| \geq 4$, there exist faces of $\mathcal{K}^{*}(N)$ which do not correspond to any $\mathcal{M}_{P}$, i.e. for $|N| \geq 4$, the set of conditional independence models is a proper sub-poset of the face poset of $\mathcal{K}^{*}(N)$.

Example 3.1 (Example 4.1 of Studený ${ }^{1}$ ). For $N=$ abcd consider

$$
\begin{aligned}
u & =u_{\langle c, d \mid a b\rangle}+u_{\langle a, b \mid \emptyset\rangle}+u_{\langle a, b \mid c\rangle}+u_{\langle a, b \mid d\rangle} \\
& =\delta_{N}+2 \cdot \delta_{a b}-\delta_{a c}-\delta_{a d}-\delta_{b c}-\delta_{b d}-\delta_{a}-\delta_{b}+\delta_{c}+\delta_{d}+\delta_{\emptyset} .
\end{aligned}
$$

Then $\langle c, d \mid a b\rangle,\langle a, b \mid \emptyset\rangle,\langle a, b \mid c\rangle,\langle a, b \mid d\rangle \in \mathcal{M}_{u}$. Furthermore let

$$
m=4 \cdot \delta_{N}+2 \cdot \sum_{S \subseteq N:|S|=3} \delta_{S}+\sum_{S \subseteq N:|S|=2, S \neq a b} \delta_{S} .
$$

(see Fig. 2.) Then it can be checked that $\langle m, v\rangle \geq 0$ for every $v \in \mathcal{E}(N)$. Hence $m$ is supermodular. Furthermore $\langle m, u\rangle=0,\left\langle m, u_{\langle a, b \mid c d\rangle}\right\rangle=1$. Consequently for any $k \in \mathbb{N},\left\langle m, k \cdot u-u_{\langle a, b \mid c d\rangle}\right\rangle=-1$ and hence $k \cdot u-$ $u_{\langle a, b \mid c d\rangle} \notin \mathcal{S}(N)$ and $\langle a, b \mid c d\rangle \notin \mathcal{M}_{u}$. On the other hand it can be shown that for any probability measure $P$ with finite multiinformation

$$
\langle c, d \mid a b\rangle,\langle a, b \mid \emptyset\rangle,\langle a, b \mid c\rangle,\langle a, b \mid d\rangle \in \mathcal{M}_{P} \Rightarrow\langle a, b \mid c d\rangle \in \mathcal{M}_{P}
$$

(cf. Corollary 2.1 of Studeny $y^{1}$ ). Therefore for the above $u$, there exists no $P$ such that $\mathcal{M}_{u}=\mathcal{M}_{P}$.

It can be shown that $m$ in (25) is an extreme ray of the supermodular cone $\mathcal{K}(N)$ for $|N|=4$ in the sense of the next section.

\section{Results on extreme rays of the supermodular cone}

In this section we prove that certain supermodular functions are "extreme rays" of the supermodular cone $\mathcal{K}(N)$. Since extreme rays of $\mathcal{K}(N)$ are hard to describe, even partial results on the extreme rays are of interest. As we discussed in Section 1. since $\mathcal{K}(N)$ contains the linear space $\mathcal{L}(N)$ of modular functions, in order to consider "extreme rays" of $\mathcal{K}(N)$ we need to identify two supermodular functions $f$ and $g$ if they differ by a modular function. For this purpose it is simplest to consider the cone $\mathcal{K}_{\ell}(N)$ of standardized supermodular functions in (6):

$$
\mathcal{K}_{\ell}(N)=\{f \in \mathcal{K}(N) \mid f(S)=0 \text { for }|S| \leq 1\},
$$

which is a pointed polyhedral cone in $\mathbb{R}^{2^{|N|}-|N|-1}$. 
Fig. 2. An extreme ray of $\mathcal{K}(N)$ not corresponding to multiinformation

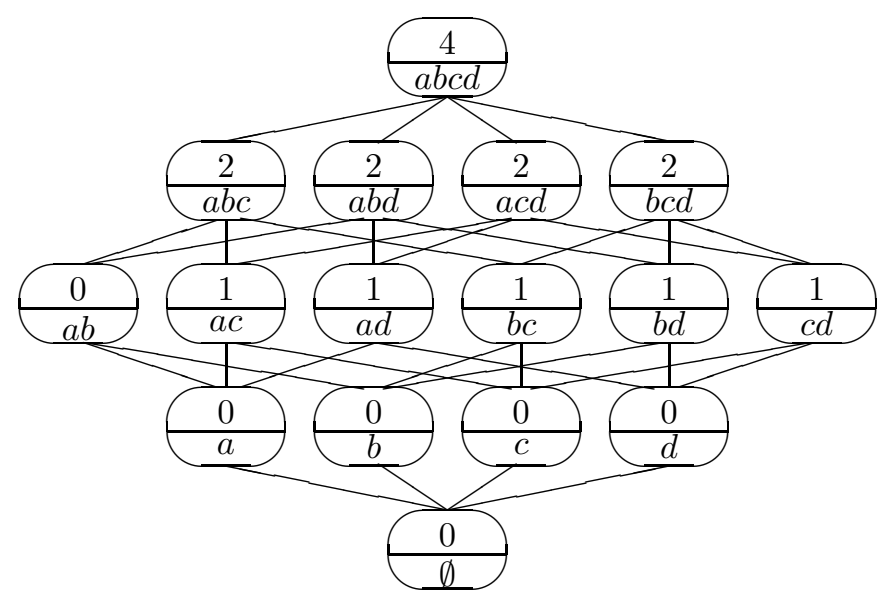

Following the terminology of Studeny ${ }^{11}$, we call an extreme ray of $\mathcal{K}_{\ell}(N)$ a skeletal supermodular function. Furthermore by abusing the terminology, we call $f \in \mathcal{K}(N)$ a skeletal supermodular function (or an extreme ray of $\mathcal{K}(N)$ ) if its standardization $\bar{f}$ in (6) is a skeletal supermodular function. Below we often omit "supermodular" and simply say that $f$ is a skeletal function, or $f$ is skeletal. Skeletal functions for $|N|=4$ are depicted in the appendix of Studený et al ${ }^{10}$ Various conditions on skeletal functions of this section are meant to be useful for understanding these functions. Except for the extreme ray $m$ in (25), other extreme rays for $|N|=4$ are covered by the results of this section. However they are far from enough for understanding most of skeletal functions for $|N|=5$ studied in Studený et al! 10

The results of this section concern extending a supermodular function on $\mathcal{P}(N)$ to a larger base set $\tilde{N} \supset N$ and resemble results in Chapter 2 of Topkis 11 , although Topkis ${ }^{11}$ does not consider skeletal functions. Results on a skeletal function as a maximum of a collection of modular functions are given in Rosenmüller and Weidner 12

For proving that a supermodular function $f$ is skeletal we proceed as in Lemma 2.1. Express $f$ as a sum of two supermodular functions

$$
f=f_{1}+f_{2} .
$$

Here we can assume that $f, f_{1}, f_{2}$ are standardized, namely, $0=f(S)=$ $f_{1}(S)=f_{2}(S)$ for $|S| \leq 1$. This follows from the fact that the standardiza- 
tion (6) can be performed to $f, f_{1}, f_{2}$ separately. We need to show that $f_{1}$ and $f_{2}$ are proportional to $f$, i.e.,

$$
f_{1}=c_{1} f, f_{2}=c_{2} f, \quad c_{1}, c_{2} \geq 0, c_{1}+c_{2}=1 .
$$

We first state two simple facts on skeletal functions. The first one concerns complementation of subsets of $N$. In Section 9.1.2 of Studeny $y^{11}$ this is referred to as a reflection. Let $A^{C}$ denote the complement of $A \subseteq N$. Let $f$ be a supermodular function. Define $f^{C}$ by

$$
f^{C}(S)=f\left(S^{C}\right), \quad S \subseteq N .
$$

Taking the complement corresponds to looking at the configuration $\mathcal{U}_{N}$ in Table 1 upside down. The following lemma is trivial.

Lemma 4.1. $f^{C}$ is skeletal if and only if $f$ is skeletal.

The second one concerns a supermodular function $f(S)$ depending only on the size $|S|$ of $S$.

Lemma 4.2. Let $|N| \geq 2$ and $1 \leq k<|N| . f(S)=\max (|S|-k, 0)$ is skeletal.

Proof. It is easily verified that $f$ is supermodular. Let $f=f_{1}+f_{2}$, where $f_{1}, f_{2}$ are standardized and supermodular. Since $f_{1}, f_{2}$ are non-negative, $f_{1}=f_{2}=0$ holds for $|S| \leq k$. This means (27) holds for $|S| \leq k$. Next we consider the case for $|S| \geq k$. If $k=|N|-1$, then (27) holds. Hence assume $k \leq|N|-2$. Let $S_{k}$ be any subset of $N$ such that $\left|S_{k}\right|=k$. Let $\tilde{S} \supset S_{k}$. Then $f(\tilde{S})=f\left(T \cup S_{k}\right)$ where $T=\tilde{S} \backslash S_{k}$. For $S_{k} \subseteq N$, define $\tilde{f}^{S_{k}}(T)=f\left(T \cup S_{k}\right)=|T|, T \subseteq N \backslash S_{k}$. In the same way, define $\tilde{f}_{i}^{S_{k}}(T)=f_{i}\left(T \cup S_{k}\right), T \subseteq N \backslash S_{k}, i=1,2$. Then $\tilde{f}^{S_{k}}$ and $\tilde{f}_{i}^{S_{k}}$ are modular function on $N \backslash S_{k}$. By (3), $\tilde{f}_{1}^{S_{k}}$ and $\tilde{f}_{2}^{S_{k}}$ can be written as

$$
\tilde{f}_{1}^{S_{k}}(T)=\lambda_{\emptyset}^{S_{k}}+\sum_{a \in T} \lambda_{a}^{S_{k}}, \quad \tilde{f}_{2}^{S_{k}}(T)=\mu_{\emptyset}^{S_{k}}+\sum_{a \in T} \mu^{S_{k}} .
$$

From $\tilde{f}^{S_{k}}(T)=|T|$ and $f=f_{1}+f_{2}$, we have $\lambda_{\emptyset}^{S_{k}}=\mu_{\emptyset}^{S_{k}}=0$ and $\lambda_{\alpha}^{S_{k}}+\mu_{\alpha}^{S_{k}}=$ 1 for $\alpha \in T$. If $\lambda_{\alpha}^{S_{k}}=\lambda_{\beta}^{S_{k}}=\lambda^{S_{k}}$ and $\mu_{\alpha}^{S_{k}}=\mu_{\beta}^{S_{k}}=\mu^{S_{k}}$ hold for all $\alpha, \beta \in N \backslash S_{k}$ where $\lambda^{S_{k}}, \mu^{S_{k}} \in \mathbb{R}^{+}$, then $\tilde{f}_{1}^{S_{k}}(T)=\lambda^{S_{k}}|T|$ and $\tilde{f}_{2}^{S_{k}}(T)=$ $\mu^{S_{k}}|T|$ hold. Furthermore we obtain the representations $\tilde{f}_{1}^{S_{k}}(T)=\lambda|T|$ and $\tilde{f}_{2}^{S_{k}}(T)=\mu|T|$ where $\lambda, \mu \in \mathbb{R}^{+}$, because $\tilde{f}_{i}^{S_{k}}\left(\alpha^{\prime}\right)=\tilde{f}_{i}^{S_{k}^{\prime}}(\alpha)$ holds if $\alpha^{\prime} S_{k}=\alpha S_{k}^{\prime}$ where $S_{k}^{\prime}$ is a subset of $N$ such that $\left|S_{k}\right|^{\prime}=k$ and $\alpha \in$ $S_{k}, \alpha^{\prime} \in S_{k}^{\prime}$. This means that (27) holds for $|S| \geq k$. It is suffices to show that $\lambda_{\alpha}^{S_{k}}=\lambda_{\beta}^{S_{k}}$ holds for all $\alpha, \beta \in N \backslash S_{k}$ such that $\alpha \neq \beta$, because we 
can prove the equation $\mu_{\alpha}^{S_{k}}=\mu_{\beta}^{S_{k}}$ in the same manner. Let $\alpha, \beta \in N \backslash S_{k}$ such that $\alpha \neq \beta$. For $\gamma \in S_{k}$, let $S_{k}^{\prime}=\{S \backslash \gamma\} \cup \alpha$ and $S_{k}^{\prime \prime}=\{S \backslash \gamma\} \cup \beta$. First we consider $\tilde{f}_{1}^{S_{k}^{\prime}}$. From $\gamma S_{k}^{\prime}=\alpha S_{k}$, we have $\tilde{f}_{1}^{S_{k}^{\prime}}(\gamma)=\tilde{f}_{1}^{S_{k}}(\alpha)=\lambda_{\alpha}^{S_{k}}$. Furthermore, from $\beta \gamma S_{k}^{\prime}=\alpha \beta S_{k}$, we have $\tilde{f}_{1}^{S_{k}^{\prime}}(\beta \gamma)=\tilde{f}_{1}^{S_{k}}(\alpha \beta)=\lambda_{\alpha}^{S_{k}}+\lambda_{\beta}^{S_{k}}$. Since $\tilde{f}_{1}^{S_{k}^{\prime}}$ is modular on $N \backslash S_{k}^{\prime}$, we obtain

$$
\tilde{f}_{1}^{S_{k}^{\prime}}(\beta)=\tilde{f}_{1}^{S_{k}^{\prime}}(\beta \gamma)+\tilde{f}_{1}^{S_{k}^{\prime}}(\emptyset)-\tilde{f}_{1}^{S_{k}^{\prime}}(\gamma)=\lambda_{\beta}^{S_{k}} .
$$

Next we consider $\tilde{f}_{1}^{S_{k}^{\prime \prime}}$. From $\gamma S_{k}^{\prime \prime}=\beta S_{k}$, we have $\tilde{f}_{1}^{S_{k}^{\prime \prime}}(\gamma)=\tilde{f}_{1}^{S_{k}}(\beta)=\lambda_{\beta}^{S_{k}}$. Furthermore, from $\alpha \gamma S_{k}^{\prime \prime}=\alpha \beta S_{k}$, we have $\tilde{f}_{1}^{S_{k}^{\prime \prime}}(\alpha \gamma)=\tilde{f}_{1}^{S_{k}}(\alpha \beta)=\lambda_{\alpha}^{S_{k}}+\lambda_{\beta}^{S_{k}}$. Since $\tilde{f}_{1}^{S_{k}^{\prime \prime}}$ is modular on $N \backslash S_{k}^{\prime \prime}$, we obtain

$$
\tilde{f}_{1}^{S_{k}^{\prime \prime}}(\alpha)=\tilde{f}_{1}^{S_{k}^{\prime \prime}}(\alpha \gamma)+\tilde{f}_{1}^{S_{k}^{\prime \prime}}(\emptyset)-\tilde{f}_{1}^{S_{k}^{\prime \prime}}(\gamma)=\lambda_{\alpha}^{S_{k}} .
$$

From (28), (29) and $\beta S_{k}^{\prime}=\alpha S_{k}^{\prime \prime}$, we have $\lambda_{\beta}^{S_{k}}=\tilde{f}_{1}^{S_{k}^{\prime}}(\beta)=\tilde{f}_{1}^{S_{k}^{\prime \prime}}(\alpha)=\lambda_{\alpha}^{S_{k}}$.

For stating other results on skeletal functions, we let $N=\{1, \ldots, n\}$, $n=|N|$, and consider $f$ as a function of $x_{1}, \ldots, x_{n}$, where $x_{i} \in\{0,1\}$, $i=1, \ldots, n . S \subseteq N$ can be identified with $\left(x_{1}, \ldots, x_{n}\right) \in\{0,1\}^{n}$, where $x_{i}=1$ if and only if $i \in S$. Let $\Delta_{i} f$ denote the function depending on $x_{j}$, $j \neq i$, defined as

$$
\Delta_{i} f=f\left(x_{1}, \ldots, x_{i-1}, 1, x_{i+1}, \ldots, x_{n}\right)-f\left(x_{1}, \ldots, x_{i-1}, 0, x_{i+1}, \ldots, x_{n}\right) .
$$

Then it is easily shown that $f$ is supermodular if and only if

$$
\Delta_{i} \Delta_{j} f \geq 0, \quad \forall i \neq j .
$$

Note that $\Delta_{i} \Delta_{j} f$ is a function of $x_{k}, k \neq i, j$. Furthermore $f$ does not depend on $x_{i}$ if and only if $\Delta_{i} f \equiv 0$. Also since a standardized supermodular function $f$ is non-decreasing, $\Delta_{i} f \geq 0$.

From now on we interchangeably write $f(S)$ or $f\left(x_{1}, \ldots, x_{n}\right)$, i.e., the argument of $f$ may be a set or a $0-1$ vector, depending on the context. We now prove the following lemma.

Lemma 4.3. Let $A \subseteq N$ and let $g: \mathcal{P}(A) \rightarrow \mathbb{R}$. Define $f: \mathcal{P}(N) \rightarrow \mathbb{R}$ by $f(S)=g(S \cap A)$. Then $f$ is supermodular if and only if $g$ is supermodular. Furthermore $f$ is skeletal if and only if $g$ is skeletal.

Proof. We omit the proof of the first statement, since it is trivial. Also it is easily shown that if $g$ is not skeletal, then $f$ is not skeletal. It remains to 
show that if $g$ is skeletal, then $f$ is skeletal. Write $f=f_{1}+f_{2}$, where $f, f_{1}, f_{2}$ are standardized. Then $0=\Delta_{j} f$ for $j \notin A$ implies $0=\Delta_{j} f_{1}=\Delta_{j} f_{2}, j \notin A$. Hence $f_{1}, f_{2}$ do not depend on $x_{j}, j \notin A$ and $f_{1}, f_{2}$ can be considered as supermodular functions on $\mathcal{P}(A)$. Then since $g$ is skeletal, $f_{1}, f_{2}$ have to be proportional to $g=f$.

For $A \subseteq N$ let

$$
f_{A}(S)=1_{A \subseteq \bullet}(S)=1_{\{A \subseteq S\}}= \begin{cases}1 & \text { if } A \subseteq S \\ 0 & \text { otherwise. }\end{cases}
$$

Using above lemmas we prove the following proposition.

Proposition 4.1. $f_{A}=1_{A \subseteq}$ is skeletal.

Proof. On $\mathcal{P}(A), g(S)=g_{A}(S)=\max (|S|-|A|+1,0)$ is skeletal by Lemma 4.2. Then its extension to $\mathcal{P}(N)$ is skeletal by Lemma 4.3 .

We now consider more complicated extensions of supermodular functions. For $f:\{0,1\}^{n} \rightarrow \mathbb{R}$ define

$$
\begin{aligned}
& f_{0}\left(x_{1}, \ldots, x_{n-1}\right)=f\left(x_{1}, \ldots, x_{n-1}, 0\right), \\
& f_{1}\left(x_{1}, \ldots, x_{n-1}\right)=f\left(x_{1}, \ldots, x_{n-1}, 1\right) .
\end{aligned}
$$

The following lemma is easy to prove.

Lemma 4.4. Let $f$ be a standardized supermodular function, such that $f_{0} \equiv 0$. Then $f$ is skeletal if and only if $f_{1}$ is skeletal.

The following result is somewhat more difficult to prove. In the lemma and its proof we denote $N^{\prime}=\{1, \ldots, n-1\}$.

Lemma 4.5. Consider $f$ such that $f_{0}$ is a skeletal function satisfying $\Delta_{i} f_{0}\left(N^{\prime} \backslash i\right)=1$ for all $i \in N^{\prime}$ and $f_{1}(S)=|S|, S \subseteq N^{\prime}$. This $f$ is skeletal.

Proof. We first show that $f$ is supermodular. It suffices to show that $\Delta_{i} \Delta_{j} f \geq 0$ for all $i, j \in N, i \neq j$. For $i, j \neq n$ and $S \subseteq N^{\prime} \backslash i j$, $\Delta_{i} \Delta_{j} f(S)=\Delta_{i} \Delta_{j} f_{0}(S) \geq 0$ because of the supermodularity of $f_{0}$. Also, for $i, j \neq n$ and $n \in S \subseteq N \backslash i j, \Delta_{i} \Delta_{j} f(S \backslash n)=\Delta_{i} \Delta_{j} f_{1}(S) \geq 0$. Thus we need to show the supermodularity when $j=n$ and $S \subseteq N^{\prime}$. From the supermodularity of $f_{0}$, for $i \in N^{\prime} \backslash S$ we have

$$
f_{0}(S i)-f_{0}(S) \leq f_{0}\left(N^{\prime}\right)-f_{0}\left(N^{\prime} \backslash i\right)=1=f_{1}(S i)-f_{1}(S),
$$


which means that

$$
\Delta_{i} \Delta_{j} f(S)=\Delta_{i}\left(f_{1}(S)-f_{0}(S)\right)=\Delta_{i} f_{1}(S)-\Delta_{i} f_{0}(S) \geq 0 .
$$

Therefore $f$ is supermodular.

Now write $f=g+h$ where $g, h$ are standardized. Define $g_{0}, g_{1}, h_{1}, h_{0}$ as above. Considering the case $x_{n}=0$, since $f_{0}$ is skeletal, there exist $c_{g}, c_{h} \geq 0, c_{g}+c_{h}=1$, such that

$$
g_{0}=c_{g} f_{0}, \quad h_{0}=c_{h} f_{0} .
$$

Also from the assumption and supermodularity of $g_{1}$ and $h_{1}$, for any $i \in N^{\prime}$ we have

$$
\begin{aligned}
& g_{1}\left(N^{\prime}\right)-g_{1}\left(N^{\prime} \backslash i\right) \geq g_{0}\left(N^{\prime}\right)-g_{0}\left(N^{\prime} \backslash i\right)=c_{g} \Delta_{i} f_{0}\left(N^{\prime} \backslash i\right)=c_{g}, \\
& h_{1}\left(N^{\prime}\right)-h_{1}\left(N^{\prime} \backslash i\right) \geq h_{0}\left(N^{\prime}\right)-h_{0}\left(N^{\prime} \backslash i\right)=c_{h} \Delta_{i} f_{0}\left(N^{\prime} \backslash i\right)=c_{h} .
\end{aligned}
$$

If at least one of the inequalities is strict, we have

$$
\begin{aligned}
1 & =(n-1)-(n-2)=f_{1}\left(N^{\prime}\right)-f_{1}\left(N^{\prime} \backslash i\right) \\
& =\left(g_{1}\left(N^{\prime}\right)-g_{1}\left(N^{\prime} \backslash i\right)\right)+\left(h_{1}\left(N^{\prime}\right)-h_{1}\left(N^{\prime} \backslash i\right)\right) \\
& >c_{g} \Delta_{i} f_{0}\left(N^{\prime} \backslash i\right)+c_{h} \Delta_{i} f_{0}\left(N^{\prime} \backslash i\right)=1,
\end{aligned}
$$

which is a contradiction. Therefore (31) and (32) are equalities.

Now consider the case that $x_{n}=1 . f_{1}(S)=|S|$ is modular. Hence both $g_{1}$ and $h_{1}$ are modular as well. From (31) and the modularity of $g_{1}$, for any $i \in N^{\prime}$ we have

$$
c_{g}=g_{1}\left(N^{\prime}\right)-g_{1}\left(N^{\prime} \backslash i\right)=g_{1}(i)-g_{1}(\emptyset)=g_{1}(i) .
$$

Furthermore from $f_{1}(\emptyset)=0$ and the modularity of $g_{1}$, we have $g_{1}(\emptyset)=0$. Hence by (3), we obtain

$$
g_{1}(S)=\sum_{i \in S} g_{1}(i)=c_{g}|S|=c_{g} f_{1}(S), S \subseteq N^{\prime} .
$$

Therefore $g=c_{g} f$. Similarly $h=c_{h} f$. This proves that $f$ is skeletal.

For our next result, we write $\boldsymbol{x}_{n-1}=\left(x_{1}, \ldots, x_{n-1}\right)$. Let $f^{\prime}:\{0,1\}^{n} \rightarrow$ $\mathbb{R}$ be supermodular. Define $f:\{0,1\}^{n+1} \rightarrow \mathbb{R}$ by

$$
f\left(\boldsymbol{x}_{n-1}, x_{n}, x_{n+1}\right)= \begin{cases}f^{\prime}\left(\boldsymbol{x}_{n-1}, 1\right) & x_{n}=x_{n+1}=1, \\ f^{\prime}\left(\boldsymbol{x}_{n-1}, 0\right) & \text { otherwise }\end{cases}
$$

Then the following result holds.

Proposition 4.2. If $f^{\prime}$ is skeletal, then $f$ is skeletal. 
Proof. As in the last proposition we write $N^{\prime}=\{1, \ldots, n-1\}$. We also write $N^{\prime \prime}=\{1, \ldots, n+1\}$. We first show that $f$ is supermodular, i.e. $\Delta_{i} \Delta_{j} f \geq 0$ for all $i, j \in N^{\prime \prime}, i \neq j$. By assumption, $\Delta_{i} \Delta_{j} f \geq 0$ for $i, j \in N^{\prime}$. Next consider the case $i \in N^{\prime}$ and $j=n+1$. Then by definition

$$
\begin{aligned}
& \Delta_{n+1} f\left(\boldsymbol{x}_{n-1}, 1\right)=\Delta_{n} f^{\prime}\left(\boldsymbol{x}_{n-1}\right), \\
& \Delta_{n+1} f\left(\boldsymbol{x}_{n-1}, 0\right)=f^{\prime}\left(\boldsymbol{x}_{n-1}, 0\right)-f^{\prime}\left(\boldsymbol{x}_{n-1}, 0\right)=0 .
\end{aligned}
$$

Hence

$$
\Delta_{i} \Delta_{n+1} f\left(\boldsymbol{x}_{n-1, \hat{i}}, 1\right)=\Delta_{i} \Delta_{n} f^{\prime}\left(\boldsymbol{x}_{n-1, \hat{i}}\right) \geq 0, \quad \Delta_{i} \Delta_{n+1} f\left(\boldsymbol{x}_{n-1, \hat{i}}, 0\right)=0,
$$

where $\boldsymbol{x}_{n-1, \hat{i}}=\boldsymbol{x}_{N^{\prime} \backslash i}$. The case of $i \in N^{\prime}, j=n$ is similar. For $i=n, j=$ $n+1$, by taking the difference of (34) and (33) we have

$$
\Delta_{n} \Delta_{n+1} f\left(\boldsymbol{x}_{n-1}\right)=\Delta_{n} f^{\prime}\left(\boldsymbol{x}_{n-1}\right) \geq 0 .
$$

Therefore $f$ is supermodular.

Now let $f=g+h$. Repeating $x_{n}$ define

$$
\tilde{f}\left(x_{1}, \ldots, x_{n}\right)=f\left(x_{1}, \ldots, x_{n}, x_{n}\right) .
$$

Then $\tilde{f}=f^{\prime}$. By assumption $\tilde{f}$ is skeletal. Hence defining $\tilde{g}, \tilde{h}$ similarly, we have

$$
\tilde{g}=c_{g} \tilde{f}, \tilde{h}=c_{h} \tilde{f}, \quad c_{g}, c_{h} \geq 0, c_{g}+c_{h}=1 .
$$

This implies that $g(x)=c_{g} f(x)$ and $h(x)=c_{h} f(x)$ if $x_{n}=x_{n+1}$. Therefore we only need to consider the cases $\left(x_{n}, x_{n+1}\right)=(1,0)$ or $\left(x_{n}, x_{n+1}\right)=(0,1)$. By symmetry we consider only the former case. Then

$$
c_{g} \tilde{f}\left(\boldsymbol{x}_{n-1}, 0\right)=\tilde{g}\left(\boldsymbol{x}_{n-1}, 0\right)=g\left(\boldsymbol{x}_{n-1}, 0,0\right) \leq g\left(\boldsymbol{x}_{n-1}, 1,0\right) .
$$

The last inequality holds by monotonicity of $g$. Similar relation holds for $h$. If at least one of the inequalities for $g$ and for $h$ is strict, then

$$
\begin{aligned}
\tilde{f}\left(\boldsymbol{x}_{n-1}, 0\right) & =c_{g} \tilde{f}\left(\boldsymbol{x}_{n-1}, 0\right)+c_{h} \tilde{f}\left(\boldsymbol{x}_{n-1}, 0\right) \\
& <g\left(\boldsymbol{x}_{n-1}, 1,0\right)+h\left(\boldsymbol{x}_{n-1}, 1,0\right)=f\left(\boldsymbol{x}_{n-1}, 1,0\right) \\
& =f^{\prime}\left(\boldsymbol{x}_{n-1}, 0\right)=\tilde{f}\left(\boldsymbol{x}_{n-1}, 0\right),
\end{aligned}
$$

which is a contradiction. Hence

$$
c_{g} f\left(\boldsymbol{x}_{n-1}, 1,0\right)=c_{g} f^{\prime}\left(\boldsymbol{x}_{n-1}, 0\right)=c_{g} \tilde{f}\left(\boldsymbol{x}_{n-1}, 0\right)=g\left(\boldsymbol{x}_{n-1}, 1,0\right)
$$

and $g=c_{g} f$. Similarly $h=c_{h} f$. Hence $f$ is skeletal. 
Our final result of this section concerns a product of skeletal functions for disjoint sets. These functions played an important role in Kashimura et al..$^{7}$

Proposition 4.3. Let $A B=N, A \cap B=\emptyset$ and consider $f$ of the form

$$
f(S)=g(A \cap S) h(B \cap S)
$$

where $g: \mathcal{P}(A) \rightarrow \mathbb{R}$ and $h: \mathcal{P}(B) \rightarrow \mathbb{R}$. Suppose that $f, g, h$ are standardized supermodular functions. Then $f$ is skeletal if and only if $g$ and $h$ are skeletal.

Note that if $g$ and $h$ are standardized supermodular functions on $\mathcal{P}(A)$ and $\mathcal{P}(B)$, respectively, then $f(S)$ in (35) is a standardized supermodular function on $\mathcal{P}(N)$.

Proof. It is easily shown that if $g$ or $h$ are not skeletal, then $f$ is not skeletal. Hence if $f$ is skeletal, then both $g$ and $h$ are skeletal.

We now show the converse. Suppose that $g$ and $h$ are skeletal. Write $f=f_{1}+f_{2}$, where $f_{1}, f_{2}$ are standardized. Consider any $A^{\prime} \subseteq A$ such that $g\left(A^{\prime}\right)>0$. For $S \subseteq B$, consider

$$
f\left(A^{\prime} S\right)=f_{1}\left(A^{\prime} S\right)+f_{2}\left(A^{\prime} S\right)=g\left(A^{\prime}\right) h(S), \quad S \subseteq B .
$$

Since $f_{1}\left(A^{\prime} S\right), f_{2}\left(A^{\prime} S\right)$ as functions of $S$ are supermodular and since $h$ is skeletal, there exist $c_{1}\left(A^{\prime}\right), c_{2}\left(A^{\prime}\right) \geq 0$, such that

$$
f_{1}\left(A^{\prime} S\right)=c_{1}\left(A^{\prime}\right) h(S), f_{2}\left(A^{\prime} S\right)=c_{2}\left(A^{\prime}\right) h(S) .
$$

Hence

$$
f\left(A^{\prime} S\right)=c_{1}\left(A^{\prime}\right) h(S)+c_{2}\left(A^{\prime}\right) h(S)=g\left(A^{\prime}\right) h(S), \quad S \subseteq B .
$$

Note that this holds also for $A^{\prime}$ such that $g\left(A^{\prime}\right)=0$ by defining $0=$ $c_{1}\left(A^{\prime}\right)=c_{2}\left(A^{\prime}\right)$. Now fixing any $S \subseteq B$ such that $h(S)>0$ and considering $f\left(A^{\prime} S\right) / h(S)=g\left(A^{\prime}\right)=c_{1}\left(A^{\prime}\right)+c_{2}\left(A^{\prime}\right)$ as a function of $A^{\prime}$, we see that $c_{1}$ and $c_{2}$ have to be proportional to $g$ because $g$ is skeletal. Then $f_{1}$ and $f_{2}$ are proportional to $f=g h$.

In Appendix A, we present a generalization of Proposition 4.3 for arbitrary cones. 


\section{Structure of faces of semi-elementary imsets}

In this section we study a face of the imset cone corresponding to a semielementary imset $u_{\langle A, B \mid C\rangle}$. By the face corresponding to $u_{\langle A, B \mid C\rangle}$, we mean the unique face of the imset cone, such that $u_{\langle A, B \mid C\rangle}$ is in the relative interior of the face. We denote this face by $F_{\langle A, B \mid C\rangle}$. In Kashimura et al! ${ }^{7}$ we have shown some remarkable facts on $F_{\langle A, B \mid C\rangle}$. Here we establish more basic facts on $F_{\langle A, B \mid C\rangle}$.

If we repeatedly apply the decomposition in (10), any semi-elementary imset $u_{\langle A, B \mid C\rangle}$ can be written as a sum of elementary imsets. Those elementary imsets are from the following set of elementary imsets:

$$
\mathcal{E}_{\langle A, B \mid C\rangle}=\left\{u_{\langle a, b \mid \Gamma\rangle} \mid a \in A, b \in B, C \subseteq \Gamma \subseteq A B C\right\} .
$$

In this section we establish the following basic facts on $F_{\langle A, B \mid C\rangle}$ : i) $\mathcal{E}_{\langle A, B \mid C\rangle}$ is the set of extreme rays of $F_{\langle A, B \mid C\rangle}$, ii $) \operatorname{dim}\left(F_{\langle A, B \mid C\rangle}\right)=\left(2^{|A|}-1\right)\left(2^{|B|}-\right.$ $1)$. For proving these facts we give linearly independent set of supermodular functions orthogonal to $F_{\langle A, B \mid C\rangle}$.

Let $D=N \backslash(A B C)$, i.e. $N=A B C D$. For $E \subseteq N$ define $1_{E \subseteq \bullet}$ by (30), which is skeletal by Proposition 4.1. Similarly define

$$
1_{E \supseteq \bullet}(S)= \begin{cases}1 & \text { if } E \supseteq S \\ 0 & \text { otherwise }\end{cases}
$$

which is skeletal by Lemma 4.1 since $1_{E \supseteq \bullet}=1_{E^{C} \subseteq \bullet}^{C}$. Now consider the following supermodular functions.

$$
\begin{array}{ll}
1_{A_{1} C \subseteq \bullet} & \left(A_{1} \subseteq A\right), \\
1_{B_{1} C \subseteq \bullet} & \left(\emptyset \neq B_{1} \subseteq B\right), \\
1_{E C_{1} \supseteq \bullet} & \left(E \subseteq A B, C_{1} \subset C\right), \\
1_{E D_{1} \subseteq \bullet} & \left(E \subseteq A B C, \emptyset \neq D_{1} \subseteq D\right) .
\end{array}
$$

Let $\mathcal{M}_{\langle A, B \mid C\rangle}$ denote the set of these supermodular functions. The cardinality of $\mathcal{M}_{\langle A, B \mid C\rangle}$ is given by

$$
\begin{aligned}
\left|\mathcal{M}_{\langle A, B \mid C\rangle}\right| & =2^{|A|}+\left(2^{|B|}-1\right)+2^{|A B|}\left(2^{|C|}-1\right)+2^{|A B C|}\left(2^{|D|}-1\right) \\
& =2^{|N|}-\left(2^{|A|}-1\right)\left(2^{|B|}-1\right) .
\end{aligned}
$$

First we check that the linear independence of the above supermodular functions.

Lemma 5.1. The elements of $\mathcal{M}_{\langle A, B \mid C\rangle}$ are linearly independent. 
Proof. We need to show that the coefficients $\lambda_{A_{1}}, \mu_{B_{1}}, \nu_{E C_{1}}, \xi_{E D_{1}} \in \mathbb{R}$ in

$$
\begin{aligned}
& \sum_{A_{1} \subseteq A} \lambda_{A_{1}} 1_{A_{1} C \subseteq \bullet}(S)+\sum_{\emptyset \neq B_{1} \subseteq B} \mu_{B_{1}} 1_{B_{1} C \subseteq \bullet}(S)+\sum_{E \subseteq A B, C_{1} \subset C} \nu_{E C_{1}} 1_{E C_{1} \supseteq} \supseteq(S) \\
& \quad+\sum_{E \subseteq A B C, \emptyset \neq D_{1} \subseteq D} \xi_{E D_{1} 1_{E D_{1} \subseteq \bullet}(S)=0 \quad(\forall S)}
\end{aligned}
$$

are zeros. Let $S=A_{2} C\left(A_{2} \subseteq A\right)$. Then $\sum_{A_{1} \subseteq A_{2}} \lambda_{A_{1}}=0$ and by induction we have $\lambda_{A_{1}}=0$. Similarly by letting $S=B_{2} C\left(\emptyset \neq B_{2} \subseteq B\right)$ we have $\sum_{\emptyset \neq B_{1} \subseteq B_{2}} \mu_{B_{1}}=0$ and by induction $\mu_{B_{1}}=0$.

Next let $S=E_{2} C_{2}\left(E_{2} \subseteq A B, C_{2} \subset C\right)$. Then

$$
\sum_{E_{2} \subseteq E \subseteq A B, C_{2} \subseteq C_{1} \subset C} \nu_{E C_{1}}=0
$$

and the double induction on $E$ and $C_{1}$ yields $\nu_{E C_{1}}=0$. Finally letting $S=E_{2} D_{2}\left(E_{2} \subseteq A B C, \emptyset \neq D_{2} \subseteq D\right)$ we have $\sum_{E \subseteq E_{2}, \emptyset \neq D_{1} \subseteq D_{2}} \xi_{E D_{1}}=0$ and the double induction on $E$ and $D_{1}$ yields $\xi_{E D_{1}}=0$.

Now we prove the following theorem.

Theorem 5.1. An elementary imset $u$ belongs to $\mathcal{E}_{\langle A, B \mid C\rangle}$ if and only if

$$
\langle u, f\rangle=0, \quad \forall f \in \mathcal{M}_{\langle A, B \mid C\rangle} .
$$

Proof. We first show the orthogonality $\langle u, f\rangle=0$ for all $u \in \mathcal{E}_{\langle A, B \mid C\rangle}$ and for all $f \in \mathcal{M}_{\langle A, B \mid C\rangle}$. Consider $f(S)=1_{A_{1} C \subseteq \bullet}\left(A_{1} \subseteq A\right)$. Let

$$
u_{\left\langle a, b \mid C^{\prime}\right\rangle}=\delta_{a b C^{\prime}}+\delta_{C^{\prime}}-\delta_{a C^{\prime}}-\delta_{b C^{\prime}} \in \mathcal{E}_{\langle A, B \mid C\rangle} .
$$

Then

$$
\begin{aligned}
\left\langle u_{\left\langle a, b \mid C^{\prime}\right\rangle}, f\right\rangle & =f\left(a b C^{\prime}\right)+f\left(C^{\prime}\right)-f\left(a C^{\prime}\right)-f\left(b C^{\prime}\right) \\
& =1_{\left\{A_{1} C \subseteq a b C^{\prime}\right\}}+1_{\left\{A_{1} C \subseteq C^{\prime}\right\}}-1_{\left\{A_{1} C \subseteq a C^{\prime}\right\}}-1_{\left\{A_{1} C \subseteq b C^{\prime}\right\}} \\
& =1_{\left\{A_{1} C \subseteq a C^{\prime}\right\}}+1_{\left\{A_{1} C \subseteq C^{\prime}\right\}}-1_{\left\{A_{1} C \subseteq a C^{\prime}\right\}}-1_{\left\{A_{1} C \subseteq C^{\prime}\right\}} \\
& =0 .
\end{aligned}
$$

Similarly $f(S)=1_{B_{1} C \subseteq \bullet}$ is orthogonal to $u_{\left\langle a, b \mid C^{\prime}\right\rangle} \in \mathcal{E}_{\langle A, B \mid C\rangle}$.

Next consider $f(S)=1_{E C_{1} \supseteq} \bullet\left(E \subseteq A B, C_{1} \subset C\right)$. Since $C \nsubseteq C_{1}$

$$
\begin{aligned}
\left\langle u_{\left\langle a, b \mid C^{\prime}\right\rangle}, f\right\rangle & =f\left(a b C^{\prime}\right)+f\left(C^{\prime}\right)-f\left(a C^{\prime}\right)-f\left(b C^{\prime}\right) \\
& =1_{\left\{E C_{1} \supseteq a b C^{\prime}\right\}}+1_{\left\{E C_{1} \supseteq C^{\prime}\right\}}-1_{\left\{E C_{1} \supseteq a C^{\prime}\right\}}-1_{\left\{E C_{1} \supseteq b C^{\prime}\right\}} \\
& =0+0-0-0=0 .
\end{aligned}
$$


Finally consider $f(S)=1_{E D_{1} \subseteq \bullet}\left(E \subseteq A B C, \emptyset \neq D_{1} \subseteq D\right)$. Then

$$
\begin{aligned}
\left\langle u_{\left\langle a, b \mid C^{\prime}\right\rangle}, f\right\rangle & =f\left(a b C^{\prime}\right)+f\left(C^{\prime}\right)-f\left(a C^{\prime}\right)-f\left(b C^{\prime}\right) \\
& =1_{\left\{E D_{1} \subseteq a b C^{\prime}\right\}}+1_{\left\{E D_{1} \subseteq C^{\prime}\right\}}-1_{\left\{E D_{1} \subseteq a C^{\prime}\right\}}-1_{\left\{E D_{1} \subseteq b C^{\prime}\right\}} \\
& =0+0-0-0=0 .
\end{aligned}
$$

We have shown that $\langle u, f\rangle=0, \forall u \in \mathcal{E}_{\langle A, B \mid C\rangle}, \forall f \in \mathcal{M}_{\langle A, B \mid C\rangle}$.

We now show that for any elementary imset $u=u_{\left\langle a^{\prime}, b^{\prime} \mid C^{\prime}\right\rangle} \notin \mathcal{E}_{\langle A, B \mid C\rangle}$ there exists $f \in \mathcal{M}_{\langle A, B \mid C\rangle}$ such that $\langle u, f\rangle=1$. For $f=1_{F \subseteq}$ we here confirm which elementary imset $u_{\langle\alpha, \beta \mid \Gamma\rangle}$ satisfies $\left\langle u_{\langle\alpha, \beta \mid \Gamma\rangle}, f\right\rangle=1$. Write

$$
\Gamma_{1}=\Gamma \cap F, \quad \Gamma_{2}=\Gamma \cap F^{C} .
$$

Then we can ignore $\Gamma_{2}$ and replace $\Gamma$ by $\Gamma_{1}$ in

$$
1_{F \subseteq \alpha \beta \Gamma}+1_{F \subseteq \Gamma}-1_{F \subseteq \alpha \Gamma}-1_{F \subseteq \beta \Gamma} .
$$

Hence we only consider $\Gamma_{1}$. It is easy to see that (42) is equal to 1 if and only if the first term of (42) is 1 and other three terms are zeros. In this case

$$
\left|\alpha \beta \Gamma_{1}\right| \geq|F|, \quad\left|\Gamma_{1}\right| \leq|F|-2
$$

implies $F=\alpha \beta \Gamma_{1}$. Putting $\Gamma_{2}$ back we have the following equivalence

$$
\begin{aligned}
\left\langle u_{\langle\alpha, \beta \mid \Gamma\rangle}, 1_{F \subseteq \bullet}\right\rangle=1 & \Leftrightarrow \alpha, \beta \in F,(\alpha \beta \Gamma) \cap F=F \\
& \Leftrightarrow \alpha, \beta \in F, \alpha \beta \Gamma \supseteq F .
\end{aligned}
$$

Similarly for $1_{F \supseteq \bullet}$ we can show

$$
\left\langle u_{\langle\alpha, \beta \mid \Gamma\rangle}, 1_{F \supseteq \bullet}\right\rangle=1 \Leftrightarrow \alpha, \beta \notin F, \Gamma \subseteq F .
$$

Now arbitrarily fix $\alpha, \beta, \Gamma$. Consider the case $\alpha \beta \Gamma \nsubseteq A B C$, i.e. $(\alpha \beta \Gamma) \cap$ $D \neq \emptyset$. Let

$$
D_{1}=(\alpha \beta \Gamma) \cap D, E=(\alpha \beta \Gamma) \cap A B C .
$$

Then $E D_{1}=\alpha \beta \Gamma$ and $\alpha, \beta \in F, \alpha \beta \Gamma \supseteq F$, where $F=E D_{1}$. Hence (43) implies $\left\langle u_{\langle\alpha, \beta \mid \Gamma\rangle}, 1_{E D_{1} \subseteq \bullet}\right\rangle=1$. It remains to consider the cases where $\alpha \beta \Gamma \subseteq A B C$. Hence assume $C \nsubseteq \Gamma \subseteq A B C$. Let $C_{1}=C \cap \Gamma$. Then $C_{1} \subset C$. Let $E=\Gamma \cap(A B)$. Then $\Gamma=E C_{1}$. For $F=\Gamma$, we have $\alpha, \beta \notin F, \Gamma=F$.

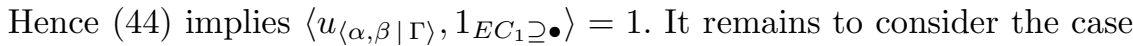
$\Gamma \supseteq C, \alpha \beta \Gamma \subseteq A B C$. Note that we want to eliminate the case that both $\alpha$ and $\beta$ belong to $A$ or belong to $B$. By symmetry we can only consider the former case. Hence assume $\alpha, \beta \in A, \Gamma \supseteq C$. In this case we can set $A_{1}=\alpha \beta(A \cap \Gamma)$. Then (43) implies $\left\langle u_{\langle\alpha, \beta \mid \Gamma\rangle}, 1_{A_{1} C \subseteq \bullet}\right\rangle=1$. 
Remark 5.1. In the above proof we have shown that

$$
\left\langle u, 1_{A \subseteq \bullet}\right\rangle,\left\langle u, 1_{A \supseteq \bullet}\right\rangle \in\{0,1\}
$$

for every elementary imset $u$ and every $A \subseteq N$.

Remark 5.2. Let $\operatorname{Lin}\left(\mathcal{M}_{\langle A, B \mid C\rangle}\right)$ and $\operatorname{Lin}\left(\mathcal{E}_{\langle A, B \mid C\rangle}\right)$ denote linear subspaces spanned by $\mathcal{M}_{\langle A, B \mid C\rangle}$ and $\mathcal{E}_{\langle A, B \mid C\rangle}$, respectively. Linearly extending (41) we see that $\operatorname{Lin}\left(\mathcal{M}_{\langle A, B \mid C\rangle}\right)$ is the orthogonal complement of $\operatorname{Lin}\left(\mathcal{E}_{\langle A, B \mid C\rangle}\right)$

Two facts stated at the beginning of this section are simple consequences of Theorem 5.1

Corollary 5.1. $\mathcal{E}_{\langle A, B \mid C\rangle}$ is the set of extreme rays of $F_{\langle A, B \mid C\rangle}$ and

$$
\operatorname{dim}\left(F_{\langle A, B \mid C\rangle}\right)=\left(2^{|A|}-1\right)\left(2^{|B|}-1\right) .
$$

The first statement is obvious and the second statement follows from Remark 5.2 and (40). The fact that $\operatorname{dim}\left(F_{\langle A, B \mid C\rangle}\right)$ does not depend on $C$ can also be seen from the one-to-one linear correspondence between $\operatorname{Lin}\left(\mathcal{E}_{\langle A, B \mid C\rangle}\right)$ and $\operatorname{Lin}\left(\mathcal{E}_{\langle A, B \mid \emptyset\rangle}\right)$ given by

$$
\mathcal{E}_{\langle A, B \mid C\rangle} \ni u_{\langle a, b \mid \Gamma\rangle} \leftrightarrow u_{\langle a, b \mid \Gamma \backslash C\rangle} \in \mathcal{E}_{\langle A, B \mid \emptyset\rangle} .
$$

Note that Theorem 5.1 shows that elements of $\mathcal{M}_{\langle A, B \mid C\rangle}$ are extreme rays of the face $F_{\langle A, B \mid C\rangle}^{\perp}$ of the supermodular cone $K(N)$ dual to $F_{\langle A, B \mid C\rangle}$. However it is hard to believe that $F_{\langle A, B \mid C\rangle}^{\perp}$ is simplicial and hence $F_{\langle A, B \mid C\rangle}^{\perp}$ should have other extreme rays than elements of $\mathcal{M}_{\langle A, B \mid C\rangle}$. Characterization of extreme rays of $F_{\langle A, B \mid C\rangle}^{\perp}$ is an interesting question left to our future research.

Remark 5.3. In our previous manuscript Kashimura et al.$^{7}$ we studied how the semi-elementary imset $u_{\langle A, B \mid C\rangle}$ is expressed as a non-negative integer combination of elements of $\mathcal{E}_{\langle A, B \mid C\rangle}$. By Theorem [5.1] if $u_{\langle A, B \mid C\rangle}$ is expressed as a non-negative integer combination of all elementary imsets, then the coefficients of $u \notin \mathcal{E}_{\langle A, B \mid C\rangle}$ have to be zero. Therefore Theorem 5.1 justifies our restriction to imsets from $\mathcal{E}_{\langle A, B \mid C\rangle}$ for expressing $u_{\langle A, B \mid C\rangle}$.

\section{Small relations among imsets}

Consider the configuration $\mathcal{U}_{N}$ as in Table 1 The kernel of $\mathcal{U}_{N}$ is denoted by ker $\mathcal{U}_{N}$. Furthermore let

$$
\operatorname{ker}_{\mathbb{Z}} \mathcal{U}_{N}=\left(\operatorname{ker} \mathcal{U}_{N}\right) \cap \mathbb{Z}^{|\mathcal{E}(N)|}
$$


denote the integer kernel of $\mathcal{U}_{N}$. Let $z=\left(z_{u}\right)_{u \in \mathcal{E}(N)} \in \mathbb{R}^{|\mathcal{E}(N)|}$ be an element of the kernel of $\mathcal{U}_{N}$. Considering $f$ in (8) as a row vector we have

$$
0=f \mathcal{U}_{N} z=(1,1, \ldots, 1) z=\sum_{u \in \mathcal{E}(N)} z_{u} .
$$

Hence any non-zero $z \in \operatorname{ker} \mathcal{U}_{N}$ has both positive and negative elements. We denote the positive elements of $z$ as $\alpha_{1}, \ldots, \alpha_{k}$ and negative elements of $z$ as $-\beta_{1}, \ldots,-\beta_{m}$. Then (45) can be written as

$$
\alpha_{1} u_{1}+\cdots+\alpha_{k} u_{k}=\beta_{1} v_{1}+\cdots+\beta_{m} v_{m},
$$

where $u_{1}, \ldots, u_{k}, v_{1}, \ldots, v_{m}$ are distinct elementary imsets. We call this kind of equality between two non-negative combinations of elementary imsets a relation among elementary imsets. More precisely we call (46) a $k$ by $m$ relation. Since $-z$ also belongs to $\operatorname{ker} \mathcal{U}_{N}$, we can take $k \leq m$. Also we can assume that $u_{1}, \ldots, u_{k}$ and $v_{1}, \ldots, v_{m}$ are ordered according to the graded reverse lexicographic order of elementary imsets. We say that the relation (46) contains another relation $\sum_{i^{\prime}=1}^{k^{\prime}} \alpha_{i^{\prime}}^{\prime} u_{i^{\prime}}^{\prime}=\sum_{j^{\prime}=1}^{m^{\prime}} \beta_{j^{\prime}}^{\prime} v_{j^{\prime}}^{\prime}$ if every $u_{i^{\prime}}^{\prime}$ appears on the left-hand side of (46) or every $v_{j^{\prime}}^{\prime}$ appears on the right-hand side of (46).

The most basic relation is the following 2 by 2 relation, which comes from the semi-graphoid axiom.

$$
u_{\left\langle a, b_{1} \mid C\right\rangle}+u_{\left\langle a, b_{2} \mid b_{1} C\right\rangle}=u_{\left\langle a, b_{2} \mid C\right\rangle}+u_{\left\langle a, b_{1} \mid b_{2} C\right\rangle} .
$$

The corresponding element of $\operatorname{ker} \mathcal{U}_{N}$ is written as

$$
\delta_{\left\langle a, b_{1} \mid C\right\rangle}+\delta_{\left\langle a, b_{2} \mid b_{1} C\right\rangle}-\delta_{\left\langle a, b_{2} \mid C\right\rangle}-\delta_{\left\langle a, b_{1} \mid b_{2} C\right\rangle},
$$

where the unit vector $\delta_{\langle a, b \mid C\rangle} \in \mathbb{Z}^{|\mathcal{E}(N)|}$ is defined as $\delta_{\langle a, b \mid C\rangle}(v)=1$ if $v=u_{\langle a, b \mid C\rangle}$ and 0 otherwise. In connection to Markov bases discussed in Section 7 we call an element of $\operatorname{ker} \mathcal{U}_{N}$ a move. Let $\mathcal{B}$ denote the collection of moves in (48) over all $a, b_{1}, b_{2}, C$. We first show that $\mathcal{B}$ generates the integer kernel of $\mathcal{U}_{N}$, i.e. $\mathcal{B}$ contains a lattice basis for $\mathcal{U}_{N}$.

Theorem 6.1. $\operatorname{ker}_{\mathbb{Z}} \mathcal{U}_{N}$ as a submodule of $\mathbb{Z}^{|\mathcal{E}(N)|}$ is generated by $\mathcal{B}$.

The following proof also shows that $\mathcal{B}$ spans $\operatorname{ker} \mathcal{U}_{N}$ as a linear subspace of $\mathbb{R}^{|\mathcal{E}(N)|}$.

Proof. Let $N=\{1, \ldots, n\}$. Let $\mathbb{Z} \mathcal{B}$ be the submodule of $\mathbb{Z}^{|\mathcal{E}(N)|}$ generated by $\mathcal{B}$. Since the inclusion $\operatorname{ker}_{\mathbb{Z}} \mathcal{U}_{N} \supseteq \mathbb{Z} \mathcal{B}$ holds, we prove $\operatorname{ker}_{\mathbb{Z}} \mathcal{U}_{N} \subseteq \mathbb{Z} \mathcal{B}$. Consider the graded reverse lexicographic order of $\mathcal{E}(N)$ in (9). Recall that 
we always assume $a<b$ if we write $u_{\langle a, b \mid C\rangle}$ and the maximum element of $\mathcal{E}(N)$ with respect to $<$ is $u_{\langle 1,2 \mid R\rangle}$, where $R=\{3, \ldots, n\}$.

For any non-zero $z \in \mathbb{Z}^{|\mathcal{E}(N)|}$ we define the $\operatorname{degree} \operatorname{deg}(z)$ by the minimum elementary imset $u_{\langle a, b \mid C\rangle}$ such that $z\left(u_{\langle a, b \mid C\rangle}\right) \neq 0$. Now fix any nonzero $z \in \operatorname{ker}_{\mathbb{Z}} \mathcal{U}_{N}$. We will find some $v \in \mathbb{Z} \mathcal{B}$ such that $\operatorname{deg}(z-v)>\operatorname{deg}(z)$ or $z=v$. Then the proposition follows by induction. We first show that $\operatorname{deg}(z)$ is not the maximum $u_{\langle 1,2 \mid R\rangle}$. Indeed, if $\operatorname{deg}(z)=u_{\langle 1,2 \mid R\rangle}$, then $z$ has to be $z\left(u_{\langle 1,2 \mid R\rangle}\right) \delta_{\langle 1,2 \mid R\rangle}$ and therefore

$$
0=\mathcal{U}_{N} \cdot z=z\left(u_{\langle 1,2 \mid R\rangle}\right) u_{\langle 1,2 \mid R\rangle},
$$

which contradicts to $z\left(u_{\langle 1,2 \mid R\rangle}\right) \neq 0$. Hence $\operatorname{deg}(z)<u_{\langle 1,2 \mid R\rangle}$. Denote $\operatorname{deg}(z)=u_{\langle a, b \mid C\rangle}$ and let $\mathcal{E}_{C}=\left\{u_{\left\langle a^{\prime}, b^{\prime} \mid C\right\rangle} \mid a^{\prime}, b^{\prime} \in N \backslash C\right\}$. Denote the maximum of $\mathcal{E}_{C}$ by $u_{\langle\alpha, \beta \mid C\rangle}$. We show that $u_{\langle a, b \mid C\rangle}<u_{\langle\alpha, \beta \mid C\rangle}$. Indeed, if $u_{\langle a, b \mid C\rangle}=u_{\langle\alpha, \beta \mid C\rangle}$, then $z$ can be written as

$$
z=z\left(u_{\langle\alpha, \beta \mid C\rangle}\right) \delta_{\langle\alpha, \beta \mid C\rangle}+\sum_{C^{\prime}>C} \sum_{a^{\prime}, b^{\prime} \in N \backslash C^{\prime}} z\left(u_{\left\langle a^{\prime}, b^{\prime} \mid C^{\prime}\right\rangle}\right) \delta_{\left\langle a^{\prime}, b^{\prime} \mid C^{\prime}\right\rangle} .
$$

Then

$$
\begin{aligned}
0=\mathcal{U}_{N} \cdot z & =z\left(u_{\langle\alpha, \beta \mid C\rangle}\right) u_{\langle\alpha, \beta \mid C\rangle}+\sum_{C^{\prime}>C} \sum_{a^{\prime}, b^{\prime} \in N \backslash C^{\prime}} z\left(u_{\left\langle a^{\prime}, b^{\prime} \mid C^{\prime}\right\rangle}\right) u_{\left\langle a^{\prime}, b^{\prime} \mid C^{\prime}\right\rangle} \\
& =z\left(u_{\langle\alpha, \beta \mid C\rangle}\right) \delta_{C}+\left(\text { terms of }\left\{\delta_{C^{\prime}}\right\}_{C^{\prime}>C}\right),
\end{aligned}
$$

which contradicts to $z\left(u_{\langle\alpha, \beta \mid C\rangle}\right) \neq 0$. Hence $u_{\langle a, b \mid C\rangle}<u_{\langle\alpha, \beta \mid C\rangle}$.

Now we give a vector $v \in \mathbb{Z} \mathcal{B}$ such that $\operatorname{deg}(z-v)>\operatorname{deg}(z)$ or $z=v$. If $b<\beta$, then consider a vector

$$
v=z\left(u_{\langle a, b \mid C\rangle}\right)\left(\delta_{\langle a, b \mid C\rangle}+\delta_{\langle a, \beta \mid b C\rangle}-\delta_{\langle a, \beta \mid C\rangle}-\delta_{\langle a, b \mid \beta C\rangle}\right)
$$

in $\mathbb{Z} \mathcal{B}$. Note that $\operatorname{deg}(v)=u_{\langle a, b \mid C\rangle}=\operatorname{deg}(z)$ and the leading terms of $v$ and $z$ are the same. Therefore we have $\operatorname{deg}(z-v)>\operatorname{deg}(z)$ or $z-v=0$. If $b=\beta$, then $a<\alpha$. Consider a vector

$$
v=z\left(u_{\langle a, b \mid C\rangle}\right)\left(\delta_{\langle a, b \mid C\rangle}+\delta_{\langle\alpha, b \mid a C\rangle}-\delta_{\langle\alpha, b \mid C\rangle}-\delta_{\langle a, b \mid \alpha C\rangle}\right)
$$

in $\mathbb{Z} \mathcal{B}$. Note that $\operatorname{deg}(v)=u_{\langle a, b \mid C\rangle}=\operatorname{deg}(z)$ and the leading terms of $v$ and $z$ are the same. Therefore we have $\operatorname{deg}(z-v)>\operatorname{deg}(z)$ or $z-v=0$. This completes the proof.

For the rest of this section we consider $k=2,3$ in (46) and show that only certain types of relations exist. By (45) we have

$$
\alpha_{1}+\cdots+\alpha_{k}=\beta_{1}+\cdots+\beta_{m}
$$


Our first result on small relations is the following.

Theorem 6.2. Consider a relation in (46) with $k=2$ :

$$
\alpha_{1} u_{1}+\alpha_{2} u_{2}=\beta_{1} v_{1}+\cdots+\beta_{m} v_{m}
$$

where $\alpha_{1}, \alpha_{2}, \beta_{1}, \ldots, \beta_{m}$ are integers. Then $m=2$ and $\alpha_{1}=\alpha_{2}=\beta_{1}=\beta_{2}$ and the relation is a positive multiple of (47).

Proof. Let $u=\alpha_{1} u_{1}+\alpha_{2} u_{2}$. From (49), we have

$$
\alpha_{1}+\alpha_{2}=\beta_{1}+\cdots+\beta_{m}, \quad \alpha_{1}>0, \alpha_{2}>0, \beta_{1}>0, \ldots, \beta_{m}>0 .
$$

Because $u_{1}, u_{2}, v_{1}, \ldots, v_{m}$ are extreme rays of $\mathcal{K}^{*}(N)$, there is no relation like $\alpha_{1} u_{1}+\alpha_{2} u_{2}=\beta_{1} v_{1}$. Therefore, we have $m \geq 2$. Furthermore, because $u_{1}, u_{2}, v_{1}, \ldots, v_{m}$ are distinct elementary imset, the following properties hold:

$$
u_{1} \neq u_{2}, \quad u_{1}, u_{2} \neq v_{1}, \ldots, v_{m}, \quad v_{i} \neq v_{j}
$$

for $i \neq j$. We write the elementary imsets in (50) as $u_{1}=u_{\left\langle a_{1}, b_{1} \mid C_{1}\right\rangle}, u_{2}=$ $u_{\left\langle a_{2}, b_{2} \mid C_{2}\right\rangle}, v_{1}=u_{\left\langle a_{1}^{\prime}, b_{1}^{\prime} \mid C_{1}^{\prime}\right\rangle}, \ldots, v_{m}=u_{\left\langle a_{m}^{\prime}, b_{m}^{\prime} \mid C_{m}^{\prime}\right\rangle}$. Remember that the terms are ordered such that $u_{1}<u_{2}$ and $v_{1}<v_{2}<\cdots<v_{m}$ according to the graded reverse lexicographic order of elementary imsets. Then there exists $l_{1} \in\{1, \ldots, m\}$ such that $C_{1}=C_{1}^{\prime}=\cdots=C_{l_{1}}^{\prime}$ and $C_{l_{1}+1}^{\prime}, \ldots, C_{m}^{\prime} \neq$ $C_{1}$. Note that if $C_{1} \neq C_{2}$, then $\alpha_{1}=\beta_{1}+\cdots+\beta_{l_{1}}$ and $a_{1} b_{1} C_{1} \neq a_{i}^{\prime} b_{i}^{\prime} C_{i}^{\prime}$ for $i \in\left\{1, \ldots, l_{1}\right\}$. In the same way, there exists $l_{2} \in\{1, \ldots, m\}$ such that

$$
a_{2} b_{2} C_{2}=a_{l_{2}}^{\prime} b_{l_{2}}^{\prime} C_{l_{2}}^{\prime}
$$

and $C_{2} \neq C_{l_{2}}^{\prime}$.

Now we define the weight $w$ for a relation as follows:

$$
w=\sum_{\{S \subseteq N} u(S)=\sum_{\left\{S \subseteq N|| S|=| C_{1} \mid+1\right\}}\left(\alpha_{1} u_{1}(S)+\alpha_{2} u_{2}(S)\right) .
$$

Then the possible values of $w$ are $-2 \alpha_{1},-2 \alpha_{1}-2 \alpha_{2}$ and $-2 \alpha_{1}+\alpha_{2}$.

Firstly, we consider the case of $w=-2 \alpha_{1}$. Then we have $\left|C_{2}\right| \geq\left|C_{1}\right|+2$. In this case, $u_{1}=v_{j}$ holds for some $j \in\{1, \ldots, m\}$ and this contradicts (51). Therefore there is no relation with $w=-2 \alpha_{1}$.

Next, we consider the case of $w=-2 \alpha_{1}-2 \alpha_{2}$. We prove $C_{1} \neq C_{2}$. If $C_{1}=C_{2}$, then by (52) there exists some $l \in\{1, \ldots, m\}$ such that $v_{l}=u_{2}$. This contradicts (51). Hence we obtain $C_{1} \neq C_{2}$. In the same way, we can prove $a_{1} b_{1} C_{1} \neq a_{2} b_{2} C_{2}$. Now, since $\left|C_{1}\right|=\left|C_{2}\right|$ in this case, we have $C_{2}=$ $C_{l_{1}+1}^{\prime}=\cdots=C_{m}^{\prime}$. However, there exists $l_{2} \in\{1, \ldots, m\}$ such that (52) holds. If $l_{2} \in\left\{l_{1}+1, \ldots, m\right\}$, then this contradicts (51). If $l_{2} \in\left\{1, \ldots, l_{1}\right\}$, 
then $C_{1} \subseteq a_{2} b_{2} C_{2}$ and $C_{2} \subseteq a_{1} b_{1} C_{1}$. This means that $C_{1}, C_{2} \subseteq a_{1} b_{1} C_{1} \cap$ $a_{2} b_{2} C_{2}$. Then we have $C_{1} \cup C_{2} \subseteq a_{1} b_{1} C_{1} \cap a_{2} b_{2} C_{2}$. From $C_{1} \neq C_{2}$ and $\left|C_{1}\right|=\left|C_{2}\right|$, we have $\left|C_{1} \cup C_{2}\right| \geq\left|C_{1}\right|+2$. Furthermore, from $a_{1} b_{1} C_{1} \neq$ $a_{2} b_{2} C_{2}$, we have $\left|a_{1} b_{1} C_{1} \cap a_{2} b_{2} C_{2}\right| \leq\left|a_{1} b_{1} C_{1}\right|-2=\left|C_{1}\right|$. However, this means that $\left|C_{1}\right|+2 \leq\left|C_{1}\right|$. This is a contradiction.

Finally, we consider the case of $w=-2 \alpha_{1}+\alpha_{2}$. Let $h=\left|C_{1}\right|$. Then we have $\left|C_{2}\right|=h+1,\left|a_{1} b_{1} C_{1}\right|=h+2,\left|a_{2} b_{2} C_{2}\right|=h+3$ and $\left|C_{i}^{\prime}\right|=h+1$ for $i \in\left\{l_{1}+1, \ldots, m\right\}$. This means that $a_{2} b_{2} C_{2}=a_{l_{1}+1}^{\prime} b_{l_{1}+1}^{\prime} C_{l_{1}+1}^{\prime}=\cdots=$ $a_{m}^{\prime} b_{m}^{\prime} C_{m}^{\prime}$. Now, assume $u\left(a_{1} b_{1} C_{1}\right) \geq 1$. Since $v_{i}(S) \leq 0$ for $|S|=h+2$ and $i \in\left\{l_{1}+1, \ldots, m\right\}$, we have $a_{1} b_{1} C_{1}=a_{j}^{\prime} b_{j}^{\prime} C_{j}^{\prime}$ for some $j \in\left\{1, \ldots, l_{1}\right\}$. This contradicts (51). Therefore $u\left(a_{1} b_{1} C_{1}\right) \leq 0$ and we have

$$
0 \geq u\left(a_{1} b_{1} C_{1}\right)=\alpha_{1} u_{1}\left(a_{1} b_{1} C_{1}\right)+\alpha_{2} u_{2}\left(a_{1} b_{1} C_{1}\right)=\alpha_{1}+\alpha_{2} u_{2}\left(a_{1} b_{1} C_{1}\right) .
$$

From the above equation, we have $u_{2}\left(a_{1} b_{1} C_{1}\right) \leq-\frac{\alpha_{1}}{\alpha_{2}}<0$. This means $u_{2}\left(a_{1} b_{1} C_{1}\right)=-1$. Therefore, we have $\alpha_{2} \geq \alpha_{1}$. In the similar way, we obtain $u\left(C_{2}\right) \leq 0$ and $\alpha_{1} \geq \alpha_{2}$. Hence we have $\alpha_{1}=\alpha_{2}$. From this, we have $u\left(C_{2}\right)=u\left(a_{1} b_{1} C_{1}\right)=0$. Then there exist $d_{1}, d_{2} \in\left\{a_{1}, b_{1}\right\}$ and $d_{3} \in\left\{a_{2}, b_{2}\right\}$ such that

$$
\begin{aligned}
C_{2} & =C_{1} \cup\left\{d_{1}\right\}, \quad a_{1} b_{1} C_{1}=C_{2} \cup\left\{d_{2}\right\}=C_{1} \cup\left\{d_{1}, d_{2}\right\}, \\
a_{2} b_{2} C_{2} & =a_{1} b_{1} C_{1} \cup\left\{d_{3}\right\}=C_{2} \cup\left\{d_{2}, d_{3}\right\}=C_{1} \cup\left\{d_{1}, d_{2}, d_{3}\right\} .
\end{aligned}
$$

Then, from $u\left(C_{1} \cup\left\{d_{2}\right\}\right)=u\left(a_{2} b_{2} C_{2} \backslash\left\{d_{2}\right\}\right)=-1$, there exist $i^{\prime} \in\left\{1, \ldots, l_{1}\right\}$ and $j^{\prime} \in\left\{l_{1}+1, \ldots, m\right\}$ such that

$$
v_{i^{\prime}}\left(C_{1} \cup\left\{d_{2}\right\}\right)=v_{j^{\prime}}\left(a_{2} b_{2} C_{2} \backslash\left\{d_{2}\right\}\right)=-1 .
$$

Let $v_{i^{\prime}}\left(C_{1} \cup d_{4}\right)=-1$ for some $d_{4} \in N \backslash\left\{C_{1} \cup d_{2}\right\}$. Then there exist $j^{\prime}$ such that $v_{j^{\prime}}\left(C_{1} \cup d_{4}\right)=1$. Since $v_{j^{\prime}}\left(a_{2} b_{2} C_{2}\right)=1$ for this $j^{\prime}$, we have $C_{1} \cup d_{4} \subseteq$ $a_{2} b_{2} C_{2}=C_{1} \cup\left\{d_{1}, d_{2}, d_{3}\right\}$. This means $d_{4} \in\left\{d_{1}, d_{3}\right\}$. Furthermore, since $v_{i^{\prime}} \neq v_{j^{\prime}}$ for $i^{\prime} \neq j^{\prime}$ in (51), $v_{i^{\prime}}$ is limited to one of $v_{i^{\prime}}=u_{\left\langle d_{2}, d_{3} \mid C_{1}\right\rangle}$ or $v_{i^{\prime}}=u_{\left\langle d_{3}, d_{2} \mid C_{1}\right\rangle}$, according to $d_{2}<d_{3}$ or $d_{3}<d_{2}$. In the same way, $v_{j^{\prime}}$ is limited to one of $u_{\left\langle d_{1}, d_{2} \mid d_{3} C_{1}\right\rangle}$ or $u_{\left\langle d_{2}, d_{1} \mid d_{3} C_{1}\right\rangle}$, according to $d_{1}<d_{2}$ or $d_{2}<d_{1}$. Therefore we obtain $m=2$. Furthermore from (53), the relation is a positive multiple of (47).

One consequence of this theorem is the following corollary on twodimensional and three-dimensional faces of $\mathcal{K}^{*}(N)$.

Corollary 6.1. Two-dimensional faces of $\mathcal{K}^{*}(N)$ are simplicial cone generated by two arbitrary elementary imsets which do not appear on the same side of the relation (47). Three-dimensional faces are either simplicial cones 
having three elementary imsets as extreme rays or a cone with four extreme rays appearing in the relation (47).

Proof. Two-dimensional cones are always simplicial. If two elementary imsets do not span a two-dimensional face, then the two-dimensional cone spanned by these two imsets cuts a relative interior of a face of a higher dimension. Then it intersects a cone generated by other extreme rays of this face. Hence these two imsets appear on one side of a 2 by $m$ relation. Then by Theorem $6.2 m=2$ and this face has to be of the form (47). If a three-dimensional cone is not simplicial, then any set of four extreme rays are linearly dependent. Hence there is a relation among them, which has to be a two by two relation. By the same argument it is easily seen that there are no more than four extreme rays of a three-dimensional face. Then by Theorem 6.2 this face has to be of the form (47).

Another important example of relation is (19) with $k=m=3$.

Theorem 6.3. Consider a relation in (46) with $k=3$ :

$$
\alpha_{1} u_{1}+\alpha_{2} u_{2}+\alpha_{3} u_{3}=\beta_{1} v_{1}+\cdots+\beta_{m} v_{m}
$$

where $\alpha_{1}, \alpha_{2}, \alpha_{3}, \beta_{1}, \ldots, \beta_{m}$ are integers. Then one of the following two properties holds.

(1) $m=3, \alpha_{1}=\alpha_{2}=\alpha_{3}=\beta_{1}=\beta_{2}=\beta_{3}$ and the relation (54) is a positive multiple of

$$
\begin{aligned}
& u_{\left\langle a, b_{1} \mid b_{2} C\right\rangle}+u_{\left\langle a, b_{2} \mid b_{3} C\right\rangle}+u_{\left\langle a, b_{3} \mid b_{1} C\right\rangle} \\
& \quad=u_{\left\langle a, b_{2} \mid b_{1} C\right\rangle}+u_{\left\langle a, b_{3} \mid b_{2} C\right\rangle}+u_{\left\langle a, b_{1} \mid b_{3} C\right\rangle}
\end{aligned}
$$

(2) The relation (54) contains a relation of Theorem 6.2

The statement in (1) can not be strengthened because there exist 3 by $m(m \geq 3)$ relations of type $(2)$ such as

$$
\begin{aligned}
2 u_{\langle a, c \mid \emptyset\rangle}+u_{\langle a, b \mid c\rangle}+u_{\langle b, c \mid a\rangle} & =u_{\langle a, b \mid \emptyset\rangle}+u_{\langle b, c \mid \emptyset\rangle}+2 u_{\langle a, c \mid b\rangle} \\
( & \left.=u_{\langle a b, c \mid \emptyset\rangle}+u_{\langle a, b c \mid \emptyset\rangle}\right),
\end{aligned}
$$

and

$$
\begin{aligned}
& u_{\left\langle a, b_{3} \mid b_{1} b_{2}\right\rangle}+2 u_{\left\langle a, b_{2} \mid b_{1}\right\rangle}+u_{\left\langle a, b_{1} \mid \emptyset\right\rangle} \\
& \quad=u_{\left\langle a, b_{2} \mid b_{1} b_{3}\right\rangle}+u_{\left\langle a, b_{3} \mid b_{1}\right\rangle}+u_{\left\langle a, b_{1} \mid b_{2}\right\rangle}+u_{\left\langle a, b_{2} \mid \emptyset\right\rangle} .
\end{aligned}
$$


Proof. Let $u=\alpha_{1} u_{1}+\alpha_{2} u_{2}+\alpha_{3} u_{3}$. From (49), we have

$\alpha_{1}+\alpha_{2}+\alpha_{3}=\beta_{1}+\cdots+\beta_{m}, \quad \alpha_{1}>0, \alpha_{2}>0, \alpha_{3}>0, \beta_{1}>0, \ldots, \beta_{m}>0$.

Because $u_{1}, u_{2}, u_{3}, v_{1}, \ldots, v_{m}$ are extreme rays of $\mathcal{K}^{*}(N)$, we have $m \geq 3$. Furthermore, because $u_{1}, u_{2}, v_{1}, \ldots, v_{m}$ are distinct elementary imsets, the following properties hold:

$$
u_{1} \neq u_{2}, \quad u_{1}, u_{2} \neq v_{1}, \ldots, v_{m}, \quad v_{i} \neq v_{j}
$$

We write the elementary imsets in (54) as $u_{1}=u_{\left\langle a_{1}, b_{1} \mid C_{1}\right\rangle}, u_{2}=$ $u_{\left\langle a_{2}, b_{2} \mid C_{2}\right\rangle}, u_{3}=u_{\left\langle a_{3}, b_{3} \mid C_{3}\right\rangle}, v_{1}=u_{\left\langle a_{1}^{\prime}, b_{1}^{\prime} \mid C_{1}^{\prime}\right\rangle}, \ldots, v_{m}=u_{\left\langle a_{m}^{\prime}, b_{m}^{\prime} \mid C_{m}^{\prime}\right\rangle}$. As in the proof of Theorem 6.2 , we define the weight $w$ for a relation as follows:

$$
w=\sum_{\left\{S \subseteq N|| S|=| C_{1} \mid+1\right\}}\left(a_{1} u_{1}(S)+a_{2} u_{2}(S)+a_{3} u_{3}(S)\right) .
$$

Remember that the terms are ordered such that $u_{1}<u_{2}<u_{3}$ and $v_{1}<v_{2}<\cdots<v_{m}$ according to the graded reverse lexicographic order of elementary imsets. Then the possible patterns are classified as follows:

(i) $w=-2\left(\alpha_{1}+\alpha_{2}+\alpha_{3}\right) \Leftrightarrow\left|C_{1}\right|=\left|C_{2}\right|=\left|C_{3}\right|$,

(ii) $w=-2\left(\alpha_{1}+\alpha_{2}\right)+\alpha_{3} \Leftrightarrow\left|C_{1}\right|=\left|C_{2}\right|=\left|C_{3}\right|-1$,

(iii) $w=-2 \alpha_{1}+\alpha_{2}+\alpha_{3} \Leftrightarrow\left|C_{1}\right|=\left|C_{2}\right|-1=\left|C_{3}\right|-1$,

(iv) $w=-2 \alpha_{1}+\alpha_{2} \Leftrightarrow\left|C_{1}\right|=\left|C_{2}\right|-1 \leq\left|C_{3}\right|-2$.

(v) $w=-2 \alpha_{1} \Leftrightarrow\left|C_{1}\right| \leq\left|C_{2}\right|-2 \leq\left|C_{3}\right|-2$.

(vi) $w=-2\left(\alpha_{1}+\alpha_{2}\right) \Leftrightarrow\left|C_{1}\right|=\left|C_{2}\right| \leq\left|C_{3}\right|-2$

First, we consider the case ( (v) of $w=-2 \alpha_{1}$. Because $u_{1} \neq v_{i}$ from (52), there exists $i^{\prime} \in\{1, \ldots m\}$ such that $v_{i^{\prime}}=\left\langle a_{1}, d^{\prime} \mid C_{1}\right\rangle, d^{\prime} \neq b_{1}$. To cancel out the value $v_{i^{\prime}}\left(d^{\prime} C_{1}\right)=-\alpha_{i^{\prime}}<0$ at $d^{\prime} C_{1}$, there exists $i^{\prime \prime} \in\{1, \ldots m\}$ such that $v_{i^{\prime \prime}}=\left\langle a^{\prime \prime}, b^{\prime \prime} \mid d^{\prime} C_{1}\right\rangle, d^{\prime} \neq b_{1}$. Furthermore there exists $d^{\prime \prime} \in\left\{a^{\prime \prime}, b^{\prime \prime}\right\}$ such that $u_{i}\left(d^{\prime} d^{\prime \prime} C_{1}\right)=0$ for $i=1,2,3$. To cancel out the value $v_{i^{\prime \prime}}\left(d^{\prime} d^{\prime \prime} C_{1}\right)=$ $-\alpha_{i^{\prime \prime}}<0$ at $d^{\prime} C_{1}$, there exists $v_{i^{\prime \prime \prime}}=\left\langle a^{\prime \prime \prime}, b^{\prime \prime \prime} \mid d^{\prime} d^{\prime \prime} C_{1}\right\rangle$. In the same way, there exists $d^{\prime \prime \prime} \in\left\{a^{\prime \prime \prime}, b^{\prime \prime \prime}\right\}$ such that $u_{i}\left(d^{\prime} d^{\prime \prime} d^{\prime \prime \prime} C_{1}\right)=0$ for $i=1,2,3$. Since it is impossible to cancel out the value $v_{i^{\prime \prime \prime}}\left(d^{\prime} d^{\prime \prime} d^{\prime \prime \prime} C_{1}\right)=-\alpha_{i^{\prime \prime \prime}}<0$ at $d^{\prime} d^{\prime \prime} d^{\prime \prime \prime} C_{1}$, this case can be ignored.

The case (vil) is almost the same as in the case of (vi).

We consider the case (iv). If $\left|C_{2}\right|-1<\left|C_{3}\right|-2$, then it contradicts (56). Therefore, we consider the case of $\left|C_{2}\right|-1=\left|C_{3}\right|-2$. In this case, there exist $m_{1}$ and $m_{2}$ such that

$$
\begin{gathered}
1 \leq m_{1} \leq m_{2} \leq m, \quad \alpha_{1}=\beta_{1}+\cdots+\beta_{m_{1}}, \quad C_{1}=C_{1}^{\prime}=\cdots=C_{m_{1}}^{\prime}, \\
\alpha_{3}=\beta_{m_{2}+1}+\cdots+\beta_{m}, \quad a_{3} b_{3} C_{3}=a_{m_{2}+1}^{\prime} b_{m_{2}+1}^{\prime} C_{m_{2}+1}^{\prime}=\cdots=a_{m}^{\prime} b_{m}^{\prime} C_{m}^{\prime}
\end{gathered}
$$


and we have $\left|C_{2}\right|=\left|C_{m_{1}+1}^{\prime}\right|=\cdots=\left|C_{m_{2}}^{\prime}\right|$. Now let $a_{1} b_{1} C_{1}=\left\{d_{1}, d_{2}\right\} \cup C_{1}$. From (56) and the same argument of the case (v), we can write $C_{2}=d_{1} C_{1}$ without loss of generality. Furthermore from (56), we have $\alpha_{1} \leq \alpha_{2}$. If $u\left(a_{1} b_{1} C_{1}\right) \leq 0$, then $\alpha_{1} u_{1}+\alpha_{2} u_{2}$ has a relation of Theorem 6.2. Therefore, we have $u\left(a_{1} b_{1} C_{1}\right)>0$. In the same way, we have $u\left(C_{3}\right)>0$. Hence there exist $i^{\prime} \in\left\{m_{2}+1, \ldots, m\right\}$ and $j^{\prime} \in\left\{1, \ldots, m_{1}\right\}$ such that $C_{i^{\prime}}^{\prime}=a_{1} b_{1} C_{1}$ and $a_{j}^{\prime} b_{j}^{\prime} C_{j}^{\prime} \in C_{3}$. This means $a_{1} b_{1} C_{1} \neq C_{3}$. Since $C_{1} \subseteq d_{1} C_{1}=C_{2} \subseteq a_{1} b_{1} C_{1}=$ $C_{i^{\prime}}^{\prime} \subseteq a_{i^{\prime}}^{\prime} b_{i^{\prime}}^{\prime} C_{i^{\prime}}^{\prime}=a_{3} b_{3} C_{3}$, we can write $a_{3} b_{3} C_{3}=d_{2} d_{3} d_{4} C_{2}=d_{1} d_{2} d_{3} d_{4} C_{1}$. From (56), we have $d_{2} C_{1} \subseteq C_{3}$. This means that $C_{3}$ is equal to $d_{2} d_{3} C_{1}$ or $d_{2} d_{4} C_{1}$. Without loss of generality, we assume that $C_{3}=d_{2} d_{3} C_{1}$. In the same way, we can show that $a_{2} b_{2} C_{2}$ is equal to $a_{3} b_{3} C_{3} \backslash d_{1}$ or $a_{3} b_{3} C_{3} \backslash d_{4}$. Since $a_{2} b_{2} C_{2} \supset C_{2}=d_{1} C_{1}$, we have $a_{2} b_{2} C_{2}=a_{3} b_{3} C_{3} \backslash d_{4}=d_{1} d_{2} d_{3} C_{1}$ and $u_{2}\left(d_{1} d_{2} C_{1}\right)=-1$. However, from $a_{1} b_{1} C_{1}=d_{1} d_{2} C_{1}$ and $a_{1} \leq a_{2}$, $u\left(a_{1} b_{1} C_{1}\right)=\alpha_{1}-\alpha_{2} \leq 0$. This is a contradiction.

Next, we consider the case (iii). If $u\left(C_{2}\right), u\left(C_{3}\right)>0$, then a contradiction follows from the proof of Theorem 6.2. Therefore, either $u\left(C_{2}\right) \leq 0$ or $u\left(C_{3}\right) \leq 0$ holds. Without loss of generality, we assume that $u\left(C_{2}\right) \leq 0$. From (56), we have $u\left(a_{1} b_{1} C_{1}\right) \leq 0$. Therefore, we obtain

$$
\begin{array}{r}
0 \geq u\left(a_{1} b_{1} C_{1}\right)=\alpha_{1}+\alpha_{2} u_{2}\left(a_{1} b_{1} C_{1}\right)+\alpha_{3} u_{3}\left(a_{1} b_{1} C_{1}\right) \\
\Longleftrightarrow \frac{\alpha_{2}}{\alpha_{1}} u_{2}\left(a_{1} b_{1} C_{1}\right)+\frac{\alpha_{3}}{\alpha_{1}} u_{3}\left(a_{1} b_{1} C_{1}\right) \leq-1 .
\end{array}
$$

From this we have $u_{2}\left(a_{1} b_{1} C_{1}\right)=-1$ or $u_{3}\left(a_{1} b_{1} C_{1}\right)=-1$. However, if we assume $u_{2}\left(a_{1} b_{1} C_{1}\right)=-1$, then $\alpha_{1} u_{1}+\alpha_{2} u_{2}$ has a relation of Theorem 6.2 Therefore, we have $u_{2}\left(a_{1} b_{1} C_{1}\right)=0$ and $u_{3}\left(a_{1} b_{1} C_{1}\right)=-1$. From this, we have $C_{2} \subseteq a_{1} b_{1} C_{1} \subseteq a_{3} b_{3} C_{3}$. In this case, if $u\left(C_{3}\right)>0$, then a contradiction follows from the proof of Theorem 6.2. Therefore $u\left(C_{3}\right) \leq 0$ holds. Furthermore, we have $u_{1}\left(C_{3}\right)=-1$. This means that $\alpha_{1} u_{1}+\alpha_{3} u_{3}$ has a relation of Theorem 6.2

The case (iii) is almost the same as in the case of (iii).

Finally, we consider the case (ii). Since $C_{i}^{\prime} \in\left\{C_{1}, C_{2}, C_{3}\right\}, a_{i}^{\prime} b_{i}^{\prime} C_{i}^{\prime} \in$ $\left\{a_{1} b_{1} C_{1}, a_{2} b_{2} C_{2}, a_{3} b_{3} C_{3}\right\}$ and (56), we have $m=3$ and $C_{i}^{\prime}=C_{i}$. In this case, $a_{1}^{\prime} b_{1}^{\prime} C_{1}^{\prime}=a_{2} b_{2} C_{2}$ or $a_{1}^{\prime} b_{1}^{\prime} C_{1}^{\prime}=a_{3} b_{3} C_{3}$ holds. Without loss of generality, we assume $a_{1}^{\prime} b_{1}^{\prime} C_{1}^{\prime}=a_{2} b_{2} C_{2}$. Then, we have $a_{2}^{\prime} b_{2}^{\prime} C_{2}^{\prime}=a_{3} b_{3} C_{3}$, $a_{3}^{\prime} b_{3}^{\prime} C_{3}^{\prime}=a_{1} b_{1} C_{1}$ and $\alpha_{1}=\alpha_{2}=\alpha_{3}=\beta_{1}=\beta_{2}=\beta_{3}$. Therefore the relation is a positive multiple of the three by three relation in (55). 


\section{Some computational results on Markov basis for imsets}

In this section we give some computational results on Markov basis for the configuration $\mathcal{U}_{N}$. Before giving them we discuss motivation for studying Markov basis from the viewpoint of inferences on conditional independence statements. In Theorem 6.1 we showed that the set of moves in (48) contains a lattice basis for $\operatorname{ker}_{\mathbb{Z}} \mathcal{U}_{N}$. This means that if we allow negative coefficients, then all relations with integer coefficients can be derived from the two by two relations of Theorem 6.2. On the other hand, recall that the three by three relation in Theorem 6.3 can not be derived from two by two relations in the sense of our discussion after (20). This is because in "applying a rule", we do not allow subtracting a non-existing term from either side of a relation. If a Markov basis is available, then we can derive any relation without ever subtracting a non-existing term starting from a given initial relation. For definitions and details of Markov basis refer to Chapter 5 of Sturmfels ${ }^{6}$ or Chapter 1 of Drton, Sturmfels and Sullivant 13

Let $\mathcal{G}\left(\mathcal{U}_{N}\right)$ be a (minimal) Markov basis for $\mathcal{U}_{N}$ and $\left|\mathcal{G}\left(\mathcal{U}_{N}\right)\right|$ be the number of elements in $\mathcal{G}\left(\mathcal{U}_{N}\right)$. If $|N| \leq 2$, then there is no relation in $\mathcal{U}_{N}$ and hence $\operatorname{ker} \mathcal{U}_{N}=\emptyset$. If $|N|=3$, then there is only the relation of (47) with $C=\emptyset$. We now give some computational results on Markov basis for $N=$ 4,5 . In the following results, we identify two relations if one is obtained by permuting characters in another. For example, we identify the following two relations: $u_{\langle a, b \mid d\rangle}+u_{\langle a, c \mid b d\rangle}=u_{\langle a, c \mid d\rangle}+u_{\langle a, b \mid c d\rangle}$ and $u_{\langle a, b \mid c\rangle}+u_{\langle b, d \mid a c\rangle}=$ $u_{\langle b, d \mid c\rangle}+u_{\langle a, b \mid c d\rangle}$. Let $\mathcal{G}^{R}\left(\mathcal{U}_{N}\right)$ be the set of representative elements in $\mathcal{G}\left(\mathcal{U}_{N}\right)$ under this equivalence relation. Let $\left|\mathcal{G}^{R}\left(\mathcal{U}_{N}\right)\right|$ be the number of elements in $\mathcal{G}^{R}\left(\mathcal{U}_{N}\right)$.

Let $\mathcal{U}_{\langle A, B \mid C\rangle}$ be a sub-configuration of $\mathcal{U}_{N}, N=A B C$, which consists of all of the elementary imsets $u_{\langle a, b \mid \Gamma\rangle}$ such that $a \in A, b \in B, C \subseteq \Gamma \subseteq A B C$, $a<b$ and $\Gamma \cap a=\Gamma \cap b=\emptyset$. Markov basis for $\mathcal{U}_{\langle A, B \mid C\rangle}$ represents the relations which appear in the face $F_{\langle A, B \mid C\rangle}$ of $\mathcal{K}^{*}(N)$. Markov bases for the subconfiguration of elementary imsets belonging to $F_{\langle A, B \mid C\rangle}$ is of some independent interest because they generate a combinatorial pure subring in the sense of Ohsugi, Herzog and Hibi ${ }^{14}\left[16\right.$. Let $\mathcal{G}^{R}(\langle A, B \mid C\rangle)$ be the representatives of Markov basis for $\mathcal{U}_{\langle A, B \mid C\rangle}$ and $\left|\mathcal{G}^{R}(\langle A, B \mid C\rangle)\right|$ be the number of elements in $\mathcal{G}^{R}(\langle A, B \mid C\rangle)$. Let $\mathcal{G}^{R}(\mathcal{T}(N))=\sum_{A B C=N} \mathcal{G}^{R}(\langle A, B \mid C\rangle)$ and $\left|\mathcal{G}^{R}(\mathcal{T}(N))\right|$ be the number of elements in $\mathcal{G}^{R}(\mathcal{T}(N))$.

For a relation of (46), we call $\alpha_{1}+\cdots+\alpha_{k}$ the degree. Note that $\sum_{i=1}^{k} \alpha_{i}=\sum_{j=1}^{m} \beta_{m}$. We give $\mathcal{G}^{R}\left(\mathcal{U}_{N}\right)$ and $\mathcal{G}^{R}(\mathcal{T}(N))$ at each degree for $|N|=4,5$ in Table 2 and 3 . The Markov basis of $\mathcal{U}_{N}$ were computed with 4 ti2 $2 \frac{17}{}$. Since the computation has not completed when we used the graded 
reverse lexicographic order in (9), we give the result for the following order:

$$
u_{\langle a, b \mid C\rangle}<u_{\left\langle a^{\prime}, b^{\prime} \mid C^{\prime}\right\rangle} \text { if }\left\{\begin{array}{l}
2^{C}>2^{C^{\prime}}, \\
\text { or } C=C^{\prime} \text { and } b>b^{\prime}, \\
\text { or } C=C^{\prime} \text { and } b=b^{\prime} \text { and } a>a^{\prime},
\end{array}\right.
$$

where $2^{C}=\sum_{i \in C} 2^{i}$.

Table 2. Number of representatives of elements of Markov basis for $|N|=4$

\begin{tabular}{ccc} 
degree & $\mathcal{G}^{R}\left(\mathcal{U}_{N}\right)$ & $\mathcal{G}^{R}(\mathcal{T}(N))$ \\
\hline 2 & 2 & 2 \\
3 & 1 & 1 \\
4 & 4 & 4
\end{tabular}

Table 3. Number of representatives of elements of Markov basis for $|N|=5$

\begin{tabular}{ccc} 
degree & $\mathcal{G}^{R}\left(\mathcal{U}_{N}\right)$ & $\mathcal{G}^{R}(\mathcal{T}(N))$ \\
\hline 2 & 3 & 3 \\
3 & 2 & 2 \\
4 & 11 & 11 \\
5 & 18 & 16 \\
6 & 210 & 162 \\
7 & 384 & 36 \\
8 & 364 & 38 \\
9 & 90 & 0 \\
10 & 220 & 0 \\
11 & 16 & 0 \\
12 & 63 & 0
\end{tabular}

In Table 2 for $|N|=4$, all of the representatives in $\mathcal{G}^{R}\left(\mathcal{U}_{N}\right)$ appear in $\mathcal{G}^{R}(\mathcal{T}(N))$. However, in Table 3 for $|N|=5$, there are many representatives which appear in $\mathcal{G}^{R}\left(\mathcal{U}_{N}\right)$ but do not appear in $\mathcal{G}^{R}(\mathcal{T}(N))$. Especially there is no relation with degree more than 8 in $\mathcal{G}^{R}(\mathcal{T}(N))$. This means that the facial structure of $\mathcal{K}^{*}(N)$ is quite complicated. Furthermore, through this computational study, though there are a lot of coefficients more than 1 in the Markov basis for $\mathcal{G}^{R}(\mathcal{T}(N))$ for $|N|=5$, we confirmed that the Markov basis for $\mathcal{G}^{R}(\langle A, B \mid C\rangle),|A|+|B|+|C| \leq 5$ is square free i.e., the coefficients in the relations are all 1 . It is future work to confirm whether this is true or not for $|N| \geq 6$. 


\section{Appendix A.}

Here we present a generalization of Proposition 4.3 to convex cones on a direct product of two finite sets.

Proposition 7.1. Let $X, Y$ be finite sets and let $G \subseteq\left\{g: X \rightarrow \mathbb{R}^{+}\right\}$, $H \subseteq\left\{h: Y \rightarrow \mathbb{R}^{+}\right\}$be pointed convex cones in $\mathbb{R}^{X}$ and $\mathbb{R}^{Y}$ respectively. Define

$$
M(G, H):=\left\{f: X \times Y \rightarrow \mathbb{R}^{+} \mid \forall x, f(x, \cdot) \in H, \forall y, f(\cdot, y) \in G\right\} .
$$

Then $M(G, H)$ is a convex cone. If $g$ and $h$ are extreme rays of $G$ and $H$, then $g(x) h(y)$ is an extreme ray of $M(G, H)$.

Proof. Let $g$ and $h$ be extreme rays of $G$ and $H$ and write $g(x) h(y)=$ $f_{1}(x, y)+f_{2}(x, y)\left(f_{1}, f_{2} \in M(G, H)\right)$. Note that there exist $x^{\prime}, y^{\prime}$ such that $g\left(x^{\prime}\right)>0$ and $h\left(y^{\prime}\right)>0$. For $x^{\prime}$ with $g\left(x^{\prime}\right)>0$ we have

$$
h(y)=\frac{f_{1}\left(x^{\prime}, y\right)}{g\left(x^{\prime}\right)}+\frac{f_{2}\left(x^{\prime}, y\right)}{g\left(x^{\prime}\right)}, \quad \forall y .
$$

Since $h$ is an extreme ray of $H$ and the two terms on the right-hand side belong to $H$ as functions of $y$ by the definition of $M(G, H), f_{1}\left(x^{\prime}, y\right)=$ $c_{1}\left(x^{\prime}\right) h(y), f_{2}\left(x^{\prime}, y\right)=c_{2}\left(x^{\prime}\right) h(y)$ for some $c_{1}\left(x^{\prime}\right), c_{2}\left(x^{\prime}\right)$. For $x$ with $g(x)=$ 0 , we have $f_{1}(x, y)=f_{2}(x, y)=0(\forall y)$ and we can put $c_{1}(x)=c_{2}(x)=0$. Then for all $x, y$ we can write $f_{1}(x, y)=c_{1}(x) h(y), f_{2}(x, y)=c_{2}(x) h(y)$. In particular for $y^{\prime}$ with $h\left(y^{\prime}\right)>0, c_{1}(x)=f_{1}\left(x, y^{\prime}\right) / h\left(y^{\prime}\right), c_{2}(x)=$ $f_{2}\left(x, y^{\prime}\right) / h\left(y^{\prime}\right)$ belong to $G$ as functions of $x$ and

$$
g(x)=\frac{f_{1}\left(x, y^{\prime}\right)+f_{2}\left(x, y^{\prime}\right)}{h\left(y^{\prime}\right)}=c_{1}(x)+c_{2}(x) .
$$

Hence $c_{1}, c_{2}$ are proportional to $g$.

We now show that Proposition 4.3 is a special case of Proposition 7.1. We note that skeletal functions are also extreme rays of $\mathcal{K}_{+}(N):=\mathcal{K}(N) \cap\{f \mid$ $f \geq 0\}$. In fact, if $f \in \mathcal{K}_{\ell}(N)$ and $f=f_{1}+f_{2}\left(f_{1}, f_{2} \in \mathcal{K}_{+}(N)\right)$, then it is easily seen that $f_{1}, f_{2} \in \mathcal{K}_{\ell}(N)$.

Let $X=2^{A}, Y=2^{B}, G=\mathcal{K}_{+}(A), H=\mathcal{K}_{+}(B)$, and let $g$ and $h$ be skeletal on $A$ and $B$. Then $g$ and $h$ are extreme rays of $G$ and $H$. Hence by Proposition $7.1 f$ is an extreme ray of $M(G, H)$. Now from the definition of supermodularity $\mathcal{K}_{\ell}(A B) \subseteq \mathcal{K}_{+}(A B) \subseteq M(G, H)$. Therefore $f$ is an extreme ray of $\mathcal{K}_{\ell}(A B)$. 
Remark 7.1. We can not put $G=\mathcal{K}_{\ell}(A), H=\mathcal{K}_{\ell}(B)$ in the above proof, because $\mathcal{K}_{\ell}(A B) \nsubseteq M(G, H)$. For example, let $f(S)=1_{\{a b \subseteq S\}}(a \in A, b \in$ $B)$, then $f \in \mathcal{K}_{\ell}(A B)$ but $f(A S)=1_{\{b \in S\}}(\forall S \subseteq B)$ does not belong to $\mathcal{K}_{\ell}(B)$ and $f \notin M\left(\mathcal{K}_{\ell}(A), \mathcal{K}_{\ell}(B)\right)$.

Acknowledgment. We are grateful to Milan Studený for very detailed and useful comments.

\section{References}

1. M. Studený, Probabilistic Conditional Independence Structures (SpringerVerlag, London, 2005).

2. M. Studený, J. Vomlel and R. Hemmecke, Internat. J. Approx. Reason. 51, $573(2010)$.

3. M. Studený and J. Vomlel, Internat. J. Approx. Reason. 52, 627 (2011).

4. B. Grünbaum, Convex Polytopes, Graduate Texts in Mathematics, Vol. 221, second edn. (Springer-Verlag, New York, 2003). Prepared and with a preface by Volker Kaibel, Victor Klee and Günter M. Ziegler.

5. T. Kashimura and A. Takemura, Standard imsets for undirected and chain graphical models arXiv:1102.2927v1, (2011).

6. B. Sturmfels, Gröbner Bases and Convex Polytopes, University Lecture Series, Vol. 8 (American Mathematical Society, Providence, RI, 1996).

7. T. Kashimura, T. Sei, A. Takemura and K. Tanaka, Journal of Algebraic Statistics 2, 14 (2011).

8. R. Hemmecke, J. Morton, A. Shiu, B. Sturmfels and O. Wienand, Combin. Probab. Comput. 17, 239 (2008).

9. M. Studený, International Journal of General System 23, 123 (1994/1995).

10. M. Studený, R. R. Bouckaert and T. Kočka, Extreme supermodular set functions over five variables Research report n. 1977, Institute of Information Theory and Automation, Prague, (2000).

11. D. M. Topkis, Supermodularity and Complementarity (Princeton University Press, Princeton, N.J., 1998).

12. J. Rosenmüller and H. G. Weidner, Discrete Math. 10, 343 (1974).

13. M. Drton, B. Sturmfels and S. Sullivant, Lectures on algebraic statistics, Oberwolfach Seminars, Vol. 39 (Birkhäuser Verlag, Basel, 2009).

14. H. Ohsugi, J. Herzog and T. Hibi, Osaka J. Math. 37, 745 (2000).

15. H. Ohsugi, Comment. Math. Univ. St. Pauli 56, 27 (2007).

16. H. Ohsugi and T. Hibi, Ann. Inst. Statist. Math. 62, 639 (2010).

17. 4ti2 team, 4ti2 - a software package for algebraic, geometric and combinatorial problems on linear spaces Available at www.4ti2.de. 